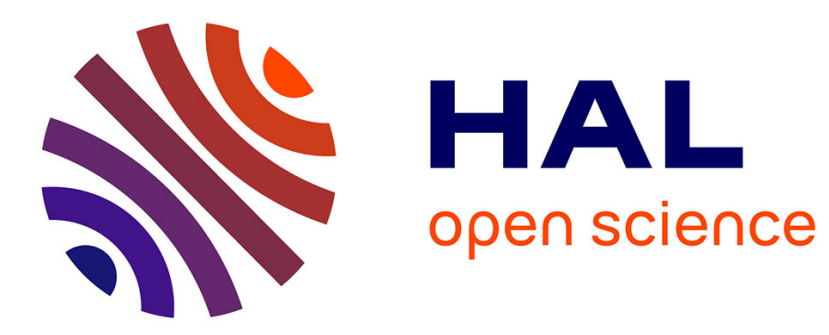

\title{
Shear modulus of simulated glass-forming model systems: Effects of boundary condition, temperature, and sampling time
}

\author{
J. P. Wittmer, H. Xu, P. Polinska, F. Weysser, J. Baschnagel
}

\section{To cite this version:}

J. P. Wittmer, H. Xu, P. Polinska, F. Weysser, J. Baschnagel. Shear modulus of simulated glassforming model systems: Effects of boundary condition, temperature, and sampling time. Journal of Chemical Physics, 2013, 138 (12), pp. 12A533. 10.1063/1.4790137 . hal-01517066

\section{HAL Id: hal-01517066 \\ https://hal.univ-lorraine.fr/hal-01517066}

Submitted on 11 May 2017

HAL is a multi-disciplinary open access archive for the deposit and dissemination of scientific research documents, whether they are published or not. The documents may come from teaching and research institutions in France or abroad, or from public or private research centers.
L'archive ouverte pluridisciplinaire HAL, est destinée au dépôt et à la diffusion de documents scientifiques de niveau recherche, publiés ou non, émanant des établissements d'enseignement et de recherche français ou étrangers, des laboratoires publics ou privés. 
Shear modulus of simulated glass-forming model systems: Effects of boundary condition, temperature, and sampling time

J. P. Wittmer', H. Xu, P. Polińska, F. Weysser, and J. Baschnagel

Citation: The Journal of Chemical Physics 138, 12 A533 (2013); doi: 10.1063/1.4790137

View online: http://dx.doi.org/10.1063/1.4790137

View Table of Contents: http://aip.scitation.org/toc/jcp/138/12

Published by the American Institute of Physics

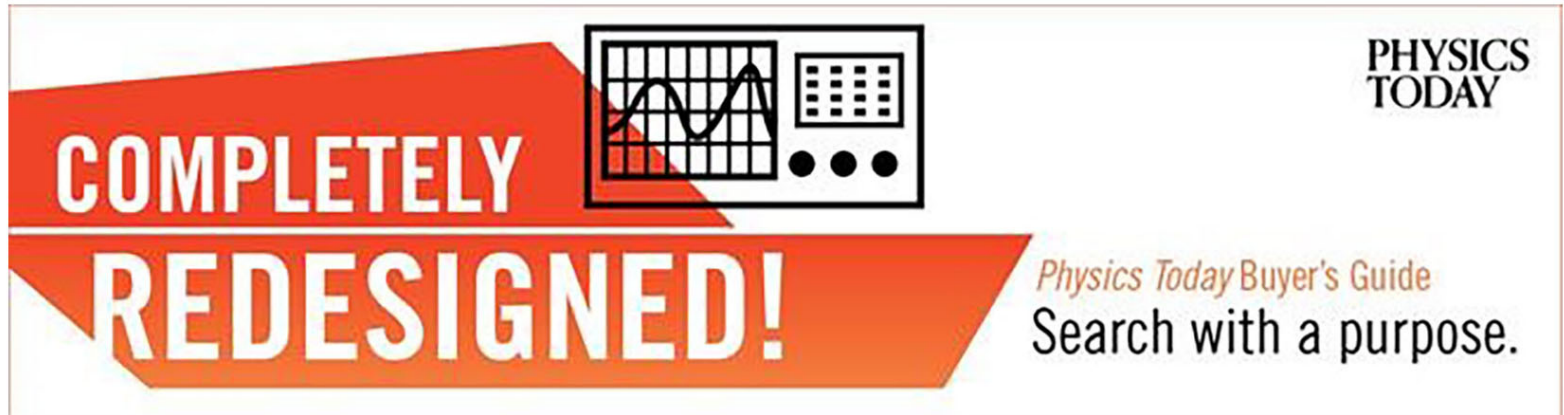




\title{
Shear modulus of simulated glass-forming model systems: Effects of boundary condition, temperature, and sampling time
}

\author{
J. P. Wittmer, ${ }^{1, a)}$ H. Xu, ${ }^{2}$ P. Polińska, ${ }^{1}$ F. Weysser, ${ }^{1}$ and J. Baschnagel ${ }^{1}$ \\ ${ }^{1}$ Institut Charles Sadron, Université de Strasbourg \& CNRS, 23 rue du Loess, 67034 Strasbourg Cedex, France \\ ${ }^{2}$ LCP-A2MC, Institut Jean Barriol, Université de Lorraine \& CNRS, 1 bd Arago, 57078 Metz Cedex 03, France
}

(Received 6 December 2012; accepted 15 January 2013; published online 11 February 2013)

\begin{abstract}
The shear modulus $G$ of two glass-forming colloidal model systems in $d=3$ and $d=2$ dimensions is investigated by means of, respectively, molecular dynamics and Monte Carlo simulations. Comparing ensembles where either the shear strain $\gamma$ or the conjugated (mean) shear stress $\tau$ are imposed, we compute $G$ from the respective stress and strain fluctuations as a function of temperature $T$ while keeping a constant normal pressure $P$. The choice of the ensemble is seen to be highly relevant for the shear stress fluctuations $\mu_{\mathrm{F}}(T)$ which at constant $\tau$ decay monotonously with $T$ following the affine shear elasticity $\mu_{\mathrm{A}}(T)$, i.e., a simple two-point correlation function. At variance, non-monotonous behavior with a maximum at the glass transition temperature $T_{\mathrm{g}}$ is demonstrated for $\mu_{\mathrm{F}}(T)$ at constant $\gamma$. The increase of $G$ below $T_{\mathrm{g}}$ is reasonably fitted for both models by a continuous cusp singularity, $G(T) \propto\left(1-T / T_{\mathrm{g}}\right)^{1 / 2}$, in qualitative agreement with recent theoretical predictions. It is argued, however, that longer sampling times may lead to a sharper transition. () 2013 American Institute of Physics. [http://dx.doi.org/10.1063/1.4790137]
\end{abstract}

\section{INTRODUCTION}

Among the fundamental properties of any solid material are its elastic constants. ${ }^{1-4}$ In their seminal work Squire, Holt and Hoover ${ }^{5}$ derived an expression for the isothermal elastic constants extending the classical Born theory ${ }^{1}$ to canonical ensembles of imposed particle number $N$, volume $V$, and finite (mean) temperature $T$. They found a correction term $C_{\mathrm{F}}^{\alpha \beta \gamma \delta}$ to the Born expression for the elastic constants which involves the mean-square fluctuations of the stress ${ }^{5-8}$

$$
C_{\mathrm{F}}^{\alpha \beta \gamma \delta} \equiv-\beta V\left(\left\langle\hat{\sigma}_{\alpha \beta} \hat{\sigma}_{\gamma \delta}\right\rangle-\left\langle\hat{\sigma}_{\alpha \beta}\right\rangle\left\langle\hat{\sigma}_{\gamma \delta}\right\rangle\right)
$$

with $\beta=1 / k_{\mathrm{B}} T$ being the inverse temperature, $k_{\mathrm{B}}$ Boltzmann's constant, $\hat{\sigma}_{\alpha \beta}$ the instantaneous value of a component of the stress tensor $\sigma_{\alpha \beta}=\left\langle\hat{\sigma}_{\alpha \beta}\right\rangle$, and greek letters denoting the spatial coordinates in $d$ dimension. Perhaps due to the fact that the demonstration of the "stress fluctuation formalism" in Ref. 5 assumed explicitly a well-defined reference position and a displacement field for the particles, it has not been appreciated that this approach is consistent (at least if the initial pressure is properly taken into account) with the well-known pressure fluctuation formula for the compression modulus $K$ of isotropic fluids ${ }^{7-10}$ which has been derived several decades earlier, presumably for the first time in the late 1940s by Rowlinson. ${ }^{9}$ In any case, the stress fluctuation formalism provides a convenient route to calculating the elastic properties in computer simulations which has been widely used in the past. $^{8,11-22}$ It has been generalized to systems with nonzero initial stress, ${ }^{11}$ hard-sphere interactions, ${ }^{13}$ and arbitrary continuous potentials, ${ }^{6}$ or to the calculation of local mechanical properties. $^{21}$

In particular from Ref. 6 it has become clear that the stress fluctuation correction does not necessarily van-

\footnotetext{
${ }^{a)}$ Electronic mail: joachim.wittmer@ics-cnrs.unistra.fr.
}

ish for $T \rightarrow 0$. This is due to the fact that the particle displacements need not follow an imposed macroscopic strain affinely. ${ }^{14-17,19,23}$ The stress fluctuation term quantifies the extent of these non-affine displacements, whereas the Born term reflects the affine part of the particle displacements. How important the non-affine motions are, depends on the system under consideration. ${ }^{19}$ While the elastic properties of crystals with one atom per unit cell are given by the Born term only, stress fluctuations are significant for crystals with more complex unit cells. ${ }^{6}$ They become pronounced for polymer like soft materials ${ }^{22}$ and amorphous solids. ${ }^{8,12,14-19,21,23,24}$ This is revealed by computational studies of various glass formers, ${ }^{12}$ such as Lennard-Jones (LJ) mixtures,${ }^{14-17}$ polymer glasses, ${ }^{8,21}$ or silica melts. ${ }^{24}$ Many of these studies concentrate on the mechanical properties deep in the glassy state, exploring for instance correlations between the non-affine displacement field and vibrational anomalies of the glass ("Boson peak"), ${ }^{17,24}$ the mechanical heterogeneity at the nanoscale, ${ }^{21,25}$ or the onset of molecular plasticity in the regime where the macroscopic deformation is still elastic. $^{26}$

Surprisingly, the temperature dependence of the elastic constants of glassy materials does not appear to have been investigated extensively using the stress fluctuation formalism. ${ }^{8,12,20}$ Following the pioneering work by Barrat et al. ${ }^{12}$ we present in this work such a study where we focus on the behavior of the shear modulus $G$ of two isotropic colloidal glass-formers in $d=3$ and $d=2$ dimensions sampled, respectively, by means of molecular dynamics (MD) and Monte Carlo (MC) simulations..$^{7,10,27}$ Since we are interested in isotropic systems one can avoid the inconvenient tensorial notation of the full stress fluctuation formalism as shown in Fig. 1. Focusing on the shear in the $x y$-plane we use $\tau \equiv \sigma_{x y}$ for the mean shear stress and $\hat{\tau} \equiv \hat{\sigma}_{x y}$ for its instantaneous value. As an important contribution to the shear modulus we 


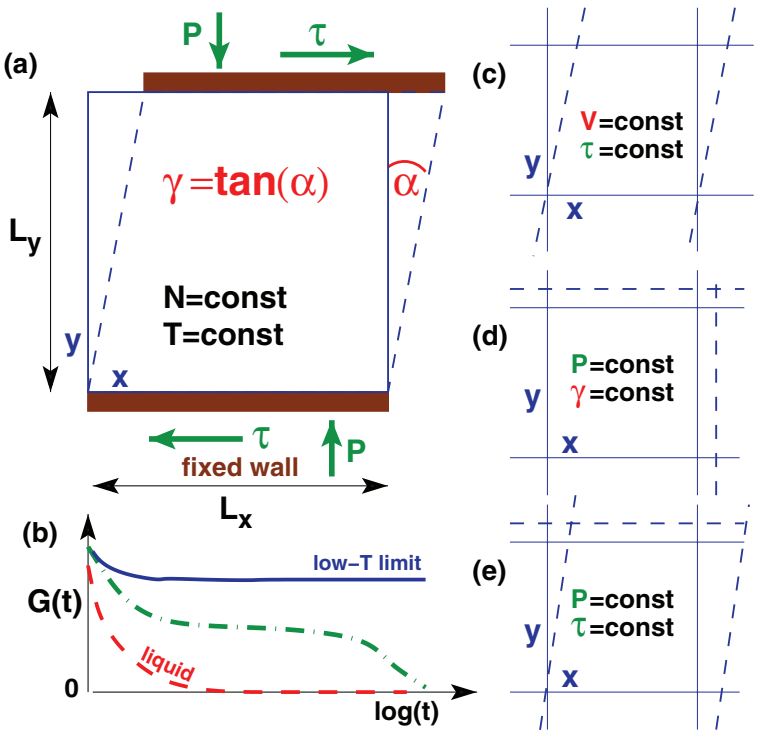

FIG. 1. Setup, notations, and ensembles investigated numerically: (a) Schematic experimental setup for probing the shear modulus $G$. (b) Expected shear modulus $G(t)$ as a function of measurement time $t$ at $T \ll T_{\mathrm{g}}$ (top curve), $T$ slightly below $T_{\mathrm{g}}$ (middle curve) and in the liquid limit for $T \gg T_{\mathrm{g}}$ (bottom curve). (c) We use periodic simulation boxes with deformable box shapes where we impose $\tau=0$. The shear modulus $G$ may be computed using Eqs. (6) or (9) from the instantaneous strains $\hat{\gamma}$ and stresses $\hat{\tau}$. (d) Using Eq. (3) $G$ may also be computed using the affine shear elasticity $\mu_{\mathrm{A}}$ and the shear stress fluctuations $\mu_{\mathrm{F}}$ in ensembles of fixed strain $(\gamma=0)$. (e) Combined volumetric and shear strain fluctuations allowing the computation of both compression modulus $K$ and shear modulus $G$ from the respective strain fluctuations.

shall monitor the shear stress fluctuations

$$
\mu_{\mathrm{F}} \equiv-C_{\mathrm{F}}^{x y x y}=\beta V\left\langle\delta \hat{\tau}^{2}\right\rangle \geq 0
$$

as a function of temperature $T$. Quite generally, the shear modulus $G$ may then be computed according to the stress fluctuation formula ${ }^{8,12}$

$$
G_{\tau \tau} \equiv \mu_{\mathrm{A}}-\mu_{\mathrm{F}},
$$

where $\mu_{\mathrm{A}}$ stands for the so-called "affine shear elasticity" corresponding to an assumed affine response to an external shear strain $\gamma$. For pairwise interaction potentials it can be shown that

$$
\mu_{\mathrm{A}} \equiv \mu_{\mathrm{B}}-P_{\mathrm{ex}} \geq 0,
$$

i.e., $\mu_{\mathrm{A}}$ comprises the so-called "Born-Lamé coefficient" $\mu_{\mathrm{B}}$, a simple moment of the first and the second derivative of the pair potential with respect to the distance of the interacting particles, ${ }^{1,8,19}$ and the excess contribution $P_{\mathrm{ex}}$ to the total pressure $P$. These notations will be properly defined in Sec. II B below where a simple derivation will be given. Since Eq. (3) is not the only operational definition of the shear modulus discussed, an index has been added reminding that the shear stress $\hat{\tau}$ is used for the determination of $G$.

If an infinitesimal shear stress $\tau$ is applied at time $t=0$, an isotropic solid exhibits, quite generally, an elastic response quantified in strength by a finite static ( $t$-independent) shear modulus $(G>0)$ as shown by the top curve in Fig. 1(b), whereas a liquid has a vanishing modulus
$(G=0)$ and flows on long time scales as shown by the bottom curve. The shear modulus $G$ can thus serve as an "order parameter" distinguishing liquid and solid states. ${ }^{28-33}$ Upon melting, the shear modulus of crystalline solids is known to vanish discontinuously with $T$. A natural question which arises is that of the behavior of $G(T)$ for amorphous solids and glasses in the vicinity of the glass transition temperature $T_{\mathrm{g}}$. Interestingly, qualitative different theoretical predictions have been put forward by mode-coupling theory (MCT) ${ }^{33,34}$ and some versions of replica theory ${ }^{32}$ predicting a discontinuous jump at the glass transition whereas other replica calculations ${ }^{30,31}$ and a very recent study of the disorder-assisted melting of amorphous solids focusing on the non-affine displacements ${ }^{23}$ suggest a continuous transition in the static limit of large sampling times $t$. (See Ref. 31 for a topical discussion of the replica approach.) The numerical characterization of $G(T)$ for colloidal glass-former is thus of high current interest. Since the stress fluctuation formalism can readily be added to the standard simulation codes of colloidal glass-formers $^{12,19,35}$ at imposed particle number $N$, box volume $V$, box shape $(\gamma=0)$ and temperature $T$, called $\mathrm{NV} \gamma \mathrm{T}$-ensembles, this suggests the use of Eq. (3) to tackle this issue. Note that the latter relation should also be useful for wide range of related systems, beyond the scope of the present work, including the glass transition of polymeric liquids,${ }^{8}$ colloidal hard sphere suspensions, ${ }^{36}$ colloidal gels,,${ }^{37}$ and self-assembled networks ${ }^{28}$ as observed experimentally, e.g., for bridged networks of telechelic polymers in water-oil emulsions. ${ }^{38}$

This begs the delicate question of whether the general stress fluctuation formalism and, especially, Eq. (3) for the shear modulus only holds for elastic solids with a well-defined microscopic displacement field, as suggested in the early literature ${ }^{5-7}$ or if the approach may actually be also applied through a solid-liquid transition up to the liquid state ${ }^{9,22}$ with $\mu_{\mathrm{F}} \rightarrow \mu_{\mathrm{A}}$ and thus $G=G_{\tau \tau} \rightarrow 0$ as it should for a liquid. By reworking the theoretical arguments leading to Eq. (3) it will be seen that this is in principal the case, at least if standard thermodynamics can be assumed to apply. The latter point is indeed not self-evident for the colloidal glasses we are interested in where (i) some degrees of freedom are frozen on the time scale probed and (ii) the measured response to an imposed infinitesimal shear $\tau$ may depend on the sampling time $t$, especially for temperatures $T$ around $T_{\mathrm{g}}$ as shown in Fig. 1(b). To be on the safer side, it thus appears to be appropriate to test numerically the applicability of the stress fluctuation formula, Eq. (3), using the corresponding strain fluctuation relations ${ }^{39,40}$ which are more fundamental on experimental grounds.

Panel (a) of Fig. 1 shows a schematic setup for probing experimentally the shear modulus $G$ of isotropic systems confined between two rigid walls. We assume that the bottom wall is fixed and the particle number $N$, the (mean) normal pressure $P$, and the (mean) temperature $T$ are kept constant. In linear response either a shear strain $\gamma=\tan (\alpha)$ or a (mean) shear stress $\tau$ may be imposed. The thin solid line indicates the unstrained reference system with $\gamma=0$ and $\tau=0$, the dashed line the deformed state. From both the mechanical and the thermodynamical point of view, the shear modulus is 
defined as

$$
\left.G \equiv \frac{\partial \tau(\gamma)}{\partial \gamma}\right|_{\gamma \rightarrow 0},
$$

where the derivative may also be taken at $\tau=0$. In practice, one obtains $G$ by fitting a linear slope to the $(\gamma, \tau)$-data at vanishing strain. Obviously, the noise level is normally large for small strains. From the numerical point of view it is thus more appropriate to work at imposed mean stress $\tau=0$, to let the strain freely fluctuate and to sample the instantaneous values $(\hat{\gamma}, \hat{\tau})$. The shear modulus $G$ is then simply obtained by linear regression

$$
\left.G_{\gamma \tau} \equiv \frac{\langle\delta \hat{\gamma} \delta \hat{\tau}\rangle}{\left\langle\delta \hat{\gamma}^{2}\right\rangle}\right|_{\tau=0}
$$

where the index indicates that both the instantaneous values $\hat{\gamma}$ and $\hat{\tau}$ are used. The associated dimensionless correlation coefficient

$$
\left.c_{\gamma \tau} \equiv \frac{\langle\delta \hat{\gamma} \delta \hat{\tau}\rangle}{\sqrt{\left\langle\delta \hat{\gamma}^{2}\right\rangle\left\langle\delta \hat{\tau}^{2}\right\rangle}}\right|_{\tau=0}
$$

allows to determine the quality of the fit. We do not know whether Eqs. (6) and (7) have actually been used in a real experiment, however since no difficulty arises to work in a computer simulation at constant $\tau$ and to record $(\hat{\gamma}, \hat{\tau})$, we use this linear regression as our key operational definition of $G$ which is used to judge the correctness of the stress fluctuation relation, Eq. (3), computed at constant $\gamma$. To avoid additional physics at the walls, we use of course periodic boundary conditions ${ }^{10}$ (the central image being sketched) as shown by the panels on the right-hand side of Fig. 1. As discussed in Sec. II C, it is possible to determine $G_{\gamma \tau}$ at constant volume (NV $\tau$ T-ensemble) as shown by panel (c) or constant normal pressure (NP $\tau$ T-ensemble) as shown by panel (e). The latter ensemble has the advantage that one can easily impose in addition the same normal pressure $P$ for all temperatures $T$ as it is justified for experimental reasons. If thermodynamics holds, it is known (Sec. II A) that

$$
\left.\langle\delta \hat{\gamma} \delta \hat{\tau}\rangle\right|_{\tau}=k_{\mathrm{B}} T / V
$$

should hold. ${ }^{41}$ Using Eq. (6) this implies a second operational definition

$$
\left.G_{\gamma \gamma} \equiv \frac{k_{\mathrm{B}} T / V}{\left\langle\delta \hat{\gamma}^{2}\right\rangle}\right|_{\tau=0},
$$

which has the advantage that only the instantaneous shear strain $\hat{\gamma}$ has to be recorded. The main technical point put forward in this review is that all operational definitions yield similar results

$$
\left.\left.\left.G_{\gamma \tau}\right|_{\tau=0} \simeq G_{\gamma \gamma}\right|_{\tau=0} \approx G_{\tau \tau}\right|_{\gamma=0},
$$

even for temperatures where a strong dependence on the sampling time $t$ is observed. In other words, Eq. (10) states that this time dependence applies similarly (albeit perhaps not exactly) to the various moments computed for the three operational definitions of $G$.

The paper is organized as follows: Several theoretical issues are regrouped in Sec. II. We begin the discussion in Sec. II A by reminding a few useful general thermodynamic relations. A simple derivation of the stress fluctuation formula, Eq. (3), for the shear modulus $G_{\tau \tau}$ in constant- $\gamma$ ensembles is given in Sec. II B. Shear stress fluctuations $\mu_{\mathrm{F}}$ in ensembles with different boundary conditions are discussed in Sec. II C. For constant- $\tau$ ensembles $\mu_{\mathrm{F}}=\left.\mu_{\mathrm{F}}\right|_{\tau}$ will be shown to reduce to the affine shear elasticity $\mu_{\mathrm{A}}$, i.e., the functional $G_{\tau \tau}$ must vanish for all temperatures. As pointed out recently, ${ }^{42}$ the truncation of the interaction potentials used in computational studies implies an impulsive correction for the affine shear elasticity $\mu_{\mathrm{A}}$. This is summarized in Sec. II D. The numerical models used in this study are presented in Sec. III. We turn then in Sec. IV to our main numerical results. Starting with the high-T liquid limit, Sec. IV A shows that all our operational definitions correctly yield a vanishing shear modulus. Moving to the opposite low- $T$ limit we present in Sec. IV B the elastic response for a topologically fixed network of harmonic springs constructed from the "dynamical matrix" of our 2D model glass system. ${ }^{14,15}$ As shown in Sec. IV C, the same behavior is found qualitatively for low$T$ glassy bead systems. The temperature dependence of stress fluctuations and shear moduli in different ensembles is then discussed in Sec. IV D. As stated above, one central goal of this work is to determine the behavior of the shear modulus around $T_{\mathrm{g}}$. We conclude the review in Sec. V. Thermodynamic relations and numerical findings related to the compression modulus $K$ are regrouped in the Appendix.

\section{THEORETICAL CONSIDERATIONS}

\section{A. Reminder of thermodynamic relations}

We assume in the following that standard thermodynamics and thermostatistics ${ }^{40}$ can be applied and remind a few useful relations. Let us denote an extensive variable by $X$ and its instantaneous value by $\hat{X}$, the conjugated intensive variable by $I$ and its instantaneous value by $\hat{I}{ }^{43}$ With $F(T, X)$ being the free energy at temperature $T$ and imposed $X$, the intensive variable $I$ and the associated modulus $M$ are given by

$$
\begin{gathered}
I \equiv \frac{\partial F(T, X)}{\partial X}, \\
M \equiv V \frac{\partial I}{\partial X}=V \frac{\partial^{2} F(T, X)}{\partial X^{2}} .
\end{gathered}
$$

In our case, the extensive variable is $X=V \gamma$, the intensive variable $I=\tau$ (Ref. 44) and the modulus $M=G$. We remind that there is an important difference between extensive and intensive variables: If the extensive variable $X$ is fixed, $\hat{X}$ cannot fluctuate. If, on the other hand, the intensive variable $I=\langle\hat{I}\rangle$ is imposed, not only $\hat{X}$ but also $\hat{I}$ may fluctuate. In our case this means, that $\hat{\gamma} \equiv \gamma$ for the $\mathrm{NV} \gamma \mathrm{T}$-ensemble, while the shear stress fluctuations $\mu_{\mathrm{F}}$ defined above do clearly not vanish in the NV $\tau$ T-ensemble shown in Fig. 1(d). Assuming $I$ to be imposed, it is well known that ${ }^{40}$

$$
\begin{gathered}
\left.\langle\delta \hat{X}(\beta \delta \hat{I})\rangle\right|_{I}=1, \\
\left.\left\langle\delta \hat{X}^{2}\right\rangle\right|_{I}=\frac{\partial X}{\partial(\beta I)}=\frac{V}{\beta M}
\end{gathered}
$$


with $\beta$ being the inverse temperature. For our variables $X=V \gamma$ and $I=\tau$, Eq. (13) implies Eq. (8) from which it is seen that Eq. (6) is consistent with Eq. (9). The latter relation is also obtained using Eq. (14).

As discussed in textbooks ${ }^{10,40}$ a simple average $A=\langle\hat{A}\rangle$ of some observable $\mathcal{A}$ does not depend on the chosen ensemble, at least not if the system is large enough $(N, V \rightarrow \infty)$. A correlation function $\langle\delta \hat{A} \delta \hat{B}\rangle$ of two observables $\mathcal{A}$ and $\mathcal{B}$ may differ, however, depending on whether $X$ or $I$ is imposed. As demonstrated by Lebowitz, Percus, and Verlet in $1967,{ }^{45}$

$$
\left.\langle\delta \hat{A} \delta \hat{B}\rangle\right|_{X}=\left.\langle\delta \hat{A} \delta \hat{B}\rangle\right|_{I}-\frac{\partial(\beta I)}{\partial X} \frac{\partial A}{\partial(\beta I)} \frac{\partial B}{\partial(\beta I)} .
$$

If the extensive variable and at least one of the observables are identical, the left-hand side of Eq. (15) vanishes: If $A=X$ and $B=I$, this yields Eq. (13). If $A=B=X$, this implies Eq. (14). More importantly, for $A=B=I$ one obtains using Eq. (12)

$$
M=\left.\beta V\left\langle\delta \hat{I}^{2}\right\rangle\right|_{I}-\left.\beta V\left\langle\delta \hat{I}^{2}\right\rangle\right|_{X} .
$$

If it is possible to probe the intensive variable fluctuations in both ensembles, this does in principle allow to determine the modulus $M$. (The latter equation might actually be taken as the definition of $M$ in cases where the thermodynamic reasoning becomes dubious.) We emphasize that the modulus $M$ is an intrinsic property of the system and does not depend on the ensemble, albeit it is determined using ensemble depending correlation functions. For a thermodynamically stable system, $M>0$, Eq. (16) implies that

$$
\left.\left\langle\delta \hat{I}^{2}\right\rangle\right|_{I}>\left.\left\langle\delta \hat{I}^{2}\right\rangle\right|_{X}
$$

and that both fluctuations must become similar in the limit where the modulus $M$ becomes small. For the shear stress fluctuations $\mu_{\mathrm{F}}$, Eq. (16) corresponds to the transformation

$$
\left.\mu_{\mathrm{F}}\right|_{V \gamma}=\left.\mu_{\mathrm{F}}\right|_{\tau}-G
$$

and one thus expects $\mu_{\mathrm{F}}$ to be larger for $\mathrm{NV} \tau \mathrm{T}$-systems than for NV $\gamma$ T-systems, while in the liquid limit where $G \rightarrow 0$ the imposed boundary conditions should not matter. We shall verify Eq. (18) numerically below. A glance at Eq. (3) suggests the identity

$$
\left.\mu_{\mathrm{F}}\right|_{\tau} \stackrel{!}{=} \mu_{\mathrm{A}}
$$

Assuming Eqs. (8) and (19), it follows for the correlation coefficient $c_{\gamma \tau}$ defined above that

$$
c_{\gamma \tau}=\sqrt{G_{\gamma \tau} /\left.\mu_{\mathrm{F}}\right|_{\tau}}=\sqrt{G / \mu_{\mathrm{A}}} .
$$

If the measured $(\hat{\gamma}, \hat{\tau})$ were indeed perfectly correlated, i.e., $c_{\gamma \tau}=1$, this implies $G=\mu_{\mathrm{A}}$ and thus $\left.\mu_{\mathrm{F}}\right|_{V \gamma}=0$. In fact, for all situations studied here we always have

$$
c_{\gamma \tau}<1, \text { thus } G<\mu_{\mathrm{A}},
$$

i.e., the affine shear elasticity sets only an upper bound to the shear modulus and a theory which only contains the affine strain response must overpredict $G$. We show now directly that Eq. (3) and, hence, Eqs. (19), (20), and (21) indeed hold this under quite general conditions.

\section{B. Shear modulus at imposed shear strain}

As shown in Fig. 1(a), we demonstrate Eq. (3) from the free energy change associated with an imposed arbitrarily small macroscopic shear strain $\gamma$ in the $x y$-plane assuming a constant particle number $N$, a constant volume $V$, and a constant temperature $T(\mathrm{NV} \gamma \mathrm{T}$-ensemble). It is supposed that the total system Hamiltonian may be written as the sum of a kinetic energy and a potential energy. Since for a plain shear strain at constant volume the ideal free energy contribution does not change, i.e., is irrelevant for $\tau$ and $G$, we may focus on the excess free energy contribution

$$
F_{\mathrm{ex}}(T, \gamma)=-k_{\mathrm{B}} T \ln \left(Z_{\mathrm{ex}}(\gamma)\right)
$$

due to the conservative interaction energy of the particles. The (excess) partition function $Z_{\mathrm{ex}}(0)$ of the unperturbed system at $\gamma=0$ is the Boltzmann-weighted sum over all states $s$ of the system which are accessible within the measurement time $t$. The argument (0) is a short-hand for the unstrained reference. Following the derivation of the compression modulus $K^{9}$ the partition function

$$
Z_{\mathrm{ex}}(\gamma)=\sum_{s} \exp \left(-\beta U_{s}(\gamma)\right)
$$

of the sheared system is supposed to be the sum over the same states $s$, but with a different metric corresponding to the macroscopic strain which changes the total interaction energy $U_{s}(\gamma)$ of state $s$ and, hence, the weight of the sheared configuration for the averages computed. This is the central hypothesis made. Interestingly, it is not necessary to specify explicitly the states of the unperturbed or perturbed system, e.g., it is irrelevant whether the particles are distinguishable or not or whether they have a well-defined reference position.

Assuming Eq. (22) we compute now the mean shear stress $\tau$ and the shear modulus $G$ for a general interaction potential $U_{s}(\gamma)$. We note for later convenience that

$$
\begin{gathered}
\frac{\partial \ln \left(Z_{\mathrm{ex}}(\gamma)\right)}{\partial \gamma}=\frac{Z_{\mathrm{ex}}^{\prime}(\gamma)}{Z_{\mathrm{ex}}(\gamma)} \\
\frac{\partial^{2} \ln \left(Z_{\mathrm{ex}}(\gamma)\right)}{\partial \gamma^{2}}=\frac{Z_{\mathrm{ex}}^{\prime \prime}(\gamma)}{Z_{\mathrm{ex}}(\gamma)}-\left(\frac{Z_{\mathrm{ex}}^{\prime}(\gamma)}{Z_{\mathrm{ex}}(\gamma)}\right)^{2}
\end{gathered}
$$

for the derivatives of the free energy and

$$
\begin{aligned}
\frac{\partial Z_{\mathrm{ex}}(\gamma)}{\partial \gamma}= & -\sum_{s} \beta U_{s}^{\prime}(\gamma) e^{-\beta U_{s}(\gamma)}, \\
\frac{\partial^{2} Z_{\mathrm{ex}}(\gamma)}{\partial \gamma^{2}}= & \sum_{s}\left(\beta U_{s}^{\prime}(\gamma)\right)^{2} e^{-\beta U_{s}(\gamma)} \\
& -\sum_{s}\left(\beta U_{s}^{\prime \prime}(\gamma)\right) e^{-\beta U_{s}(\gamma)}
\end{aligned}
$$

for the derivatives of the excess partition function where a prime denotes the derivative of a function $f(x)$ with respect to its argument $x$. Using Eq. (26) and taking the limit $\gamma \rightarrow 0$ one verifies for the shear stress that

$$
\tau=\langle\hat{\tau}\rangle \quad \text { with }\left.\hat{\tau} \equiv \frac{1}{V} U_{s}^{\prime}(\gamma)\right|_{\gamma=0}
$$


defining the instantaneous shear stress. ${ }^{44}$ Note that the average taken is defined as

$$
\langle\ldots\rangle=\frac{1}{Z_{\mathrm{ex}}(0)} \sum_{s} \ldots e^{-\beta U_{s}(0)}
$$

using the weights of the unperturbed system. The shear stress thus measures the average change of the total interaction energy $U_{s}(\gamma)$ taken at $\gamma=0$. The shear modulus $G$ is obtained using in addition Eqs. (25) and (27) and taking finally the $\gamma \rightarrow 0$ limit. Confirming thus the stress fluctuation formula Eq. (3), this yields

$$
G=G_{\tau \tau} \equiv \mu_{\mathrm{A}}-\left.\mu_{\mathrm{F}}\right|_{\gamma=0}
$$

with $\mu_{\mathrm{A}}$ being the "affine shear elasticity"

$$
\mu_{\mathrm{A}} \equiv \frac{1}{V}\left\langle\left. U_{s}^{\prime \prime}(\gamma)\right|_{\gamma=0}\right\rangle
$$

mentioned above and $\mu_{\mathrm{F}}$ as defined in Eq. (2). The comparison of Eqs. (18) and (30) confirms Eq. (19). The affine shear elasticity $\mu_{\mathrm{A}}$ corresponds to the change (second derivative) of the total energy which would be obtained if one actually strains affinely in a computer simulation a given state $s$ without allowing the particles to relax their position. It is for this reason that the shear-stress fluctuation term $\mu_{\mathrm{F}} \geq 0$ must occur in Eq. (30) correcting the overprediction.

Up to now we have not used the coordinate transformation (metric change) associated with an affine shear strain. This is needed to obtain operational definitions for the instantaneous shear stress $\hat{\tau}$ (and thus for $\tau$ and $\mu_{\mathrm{F}}$ ) and the affine shear elasticity. As shown in Fig. 1(a), a pure shear strain transforms the $x$-coordinate of a particle position or the distance between particles as

$$
x(0) \Rightarrow x(\gamma)=x(0)+\gamma y(0)
$$

leaving all other coordinates unchanged. The squared particle distance $r^{2}$ thus transforms as

$$
r^{2}(0) \Rightarrow r^{2}(\gamma)=r^{2}(0)+2 \gamma x(0) y(0)+\gamma^{2} y^{2}(0),
$$

where the $\gamma^{2}$-term is required for the calculation of the shear modulus below. This implies

$$
\begin{gathered}
\frac{d r^{2}(\gamma)}{d \gamma}=2 x(0) y(0)+2 \gamma y^{2}(0), \\
\frac{d^{2} r^{2}(\gamma)}{d \gamma^{2}}=2 y^{2}(0)
\end{gathered}
$$

for, respectively, the first and the second derivative with respect to $\gamma$. Let us now consider an arbitrary function $f(r(\gamma))$. Using Eq. (34) it is readily seen that its first derivative with respect to $\gamma$ may be written as

$$
\begin{aligned}
\frac{\partial f(r(\gamma))}{\partial \gamma} & =\frac{f^{\prime}(r)}{2 r(0)}\left(2 x(0) y(0)+2 \gamma y^{2}(0)\right) \\
& \Rightarrow r f^{\prime}(r) n_{x} n_{y} \text { for } \gamma \rightarrow 0 .
\end{aligned}
$$

In the second step we have introduced the components $n_{x}$ $=x(0) / r(0)$ and $n_{y}=y(0) / r(0)$ of the normalized distance vector. More generally, we denote by $n_{\alpha}$ the $\alpha$-component of a normalized vector with $\alpha=x, y, \ldots$ Stating only the small- $\gamma$ limit for the second derivative of $f(r(\gamma))$ with respect to $\gamma$ we note finally that

$$
\begin{aligned}
\lim _{\gamma \rightarrow 0}\left(\frac{\partial^{2} f(r(\gamma))}{\partial \gamma^{2}}\right)= & \left(r^{2} f^{\prime \prime}(r)-r f^{\prime}(r)\right) n_{x}^{2} n_{y}^{2} \\
& +r f^{\prime}(r) n_{y} n_{y},
\end{aligned}
$$

where we have dropped the argument (0) for the distance $r$ of the unperturbed reference system.

We assume now that the interaction energy $U_{s}(\gamma)$ of a configuration $s$ is given by a pair interaction potential $u(r)$ between the particles ${ }^{7,10}$ with $r(\gamma)$ being the distance between two particles, i.e.,

$$
U_{s}(\gamma)=\sum_{l} u\left(r_{l}(\gamma)\right)
$$

where the index $l$ labels the interaction between the particles $i$ and $j$ with $i<j$. Using the general result Eq. (28) for the shear stress it follows using Eq. (36) that

$$
\hat{\tau}=\frac{1}{V} \sum_{l} r_{l} u^{\prime}\left(r_{l}\right) n_{x, l} n_{y, l}
$$

where we have dropped the argument (0) in the $\gamma \rightarrow 0$ limit. We have thus rederived for the shear stress $(\alpha=x, \beta=y)$ the well-known Kirkwood expression for the general excess stress tensor ${ }^{10}$

$$
\sigma_{\alpha \beta}^{\mathrm{ex}}=\left\langle\hat{\sigma}_{\alpha \beta}^{\mathrm{ex}}\right\rangle \quad \text { with } \hat{\sigma}_{\alpha \beta}^{\mathrm{ex}} \equiv \frac{1}{V} \sum_{l} r_{l} u^{\prime}\left(r_{l}\right) n_{\alpha, l} n_{\beta, l} .
$$

The affine shear elasticity $\mu_{\mathrm{A}}$ is obtained using Eqs. (31) and (38). This yields

$$
\mu_{\mathrm{A}}=\mu_{\mathrm{B}}+\sigma_{y y}^{\mathrm{ex}}
$$

with the first contribution $\mu_{\mathrm{B}}$ being the so-called "Born-Lamé coefficient"1

$$
\mu_{\mathrm{B}} \equiv \frac{1}{V}\left\langle\sum_{l}\left(r_{l}^{2} u^{\prime \prime}\left(r_{l}\right)-r_{l} u^{\prime}\left(r_{l}\right)\right) n_{x, l}^{2} n_{y, l}^{2}\right\rangle .
$$

It corresponds to the first term in Eq. (38).

In this work we focus on isotropic materials under isotropic external loads. The normal excess stresses $\sigma_{\alpha \alpha}^{\text {ex }}$ for all $\alpha$ are thus identical, i.e.

$$
\sigma_{\alpha \alpha}^{\mathrm{ex}} \stackrel{!}{=}-P_{\mathrm{ex}}=\frac{1}{d V}\left\langle\sum_{l} r_{l} u^{\prime}\left(r_{l}\right)\right\rangle
$$

with $P_{\text {ex }} \equiv P-P_{\text {id }}$ being the excess pressure, $P$ the total pressure, $P_{\mathrm{id}}=k_{\mathrm{B}} T \rho$ the ideal gas pressure, and $d$ the spatial dimension. This implies that the affine shear elasticity is given by $\mu_{\mathrm{A}}=\mu_{\mathrm{B}}-P_{\mathrm{ex}}$ as already stated in the Introduction, Eq. (4). We note that for isotropic $d$-dimensional systems it can be shown ${ }^{22,42}$ that the Born-Lamé coefficient can be simplified as

$$
\mu_{\mathrm{B}}=\frac{1}{d(d+2) V} \sum_{l}\left\langle r_{l}^{2} u^{\prime \prime}\left(r_{l}\right)-r_{l} u^{\prime}\left(r_{l}\right)\right\rangle .
$$


The $d$-dependent prefactor stems from the assumed isotropy of the system and the mathematical formula

$$
\left\langle\left(n_{\alpha} n_{\beta}\right)^{2}\right\rangle=\frac{1}{d(d+2)}\left(1+2 \delta_{\alpha \beta}\right)
$$

$\left(\delta_{\alpha \beta}\right.$ being the Kronecker symbol $\left.{ }^{46}\right)$ for the components of a unit vector in $d$ dimensions pointing into arbitrary directions. By comparing the excess pressure $P_{\text {ex }}$, Eq. (44), with the underlined contribution to the Born coefficient in Eq. (45) this implies

$$
\frac{-1}{d(d+2) V}\left\langle\sum_{l} r_{l} u^{\prime}\left(r_{l}\right)\right\rangle=P_{\mathrm{ex}} /(d+2) .
$$

It is thus inconsistent to neglect the explicit excess pressure in Eq. (4) while keeping the second term of the Born-Lamé coefficient $\mu_{\mathrm{B}}$. This approximation is only justified if the excess pressure $P_{\mathrm{ex}}$ is negligible.

\section{Shear stress fluctuations in different ensembles}

Being simple averages neither $P_{\mathrm{ex}}, \mu_{\mathrm{A}}$ nor $\mu_{\mathrm{B}}$ depend for sufficiently large systems on the chosen ensemble, i.e., irrespective of whether $\gamma$ or $\tau$ is imposed one expects to obtain the same values. As already reminded in Sec. II A, this is different for fluctuations in general. ${ }^{10}$ For instance, it is obviously pointless to use the shear-strain fluctuation formulae $G_{\gamma \tau}$ or $G_{\gamma \gamma}$ in a constant- $\gamma$ ensemble. A more interesting result is predicted if $G_{\tau \tau} \equiv \mu_{\mathrm{A}}-\mu_{\mathrm{F}}$ is computed in the "wrong" constant $\tau$ ensemble. Since $\left.\mu_{\mathrm{F}}\right|_{\tau}=\mu_{\mathrm{A}}$, Eq. (18), one actually expects to observe $G_{\tau \tau}=0$ for all temperatures $T$ if our thermodynamic reasoning holds through the glass transition up to the liquid state. We will test numerically this non-trivial prediction in Sec. IV.

Up to now we have only considered for simplicity of the presentation $\mathrm{NV} \gamma \mathrm{T}$ - and $\mathrm{NV} \tau \mathrm{T}$-ensembles at constant volume $V$. Since on general grounds pure deviatoric and dilatational (volumetric) strains are decoupled (both strains commute), one expects the observable $G_{\tau \tau}$ also to be applicable in the NP $\gamma$ T-ensemble shown in Fig. 1(d) and the observables $G_{\gamma \tau}$ and $G_{\gamma \gamma}$ to be applicable in the NP $\tau$ T-ensemble as sketched in Fig. 1(e). In the latter ensemble one also expects to find $G_{\tau \tau}=0$ and $\left.\mu_{\mathrm{F}}\right|_{\tau}=\mu_{\mathrm{A}}$. This will also be tested below.

We demonstrate now directly Eq. (19) using the general mathematical identity

$$
\langle f(x) A(x)\rangle=-k_{\mathrm{B}} T\left\langle A^{\prime}(x)\right\rangle
$$

with $x$ being an unconstrained coordinate, $A(x)$ some property, $f(x) \equiv-u^{\prime}(x)$ a "force" with respect to some "energy" $u(x)$ and the average being Boltzmann weighted, i.e., $\langle\ldots\rangle$ $\propto \int \mathrm{d} x \ldots e^{-\beta u(x)}$. (It is assumed in Eq. (48) that $u(x)$ decays sufficiently fast at the boundaries.) Following Zwanzig, ${ }^{47}$ the instantaneous shear stress $\hat{\tau}$ is expressed by the Kirkwood formula, Eq. (40), by the alternative virial representation ${ }^{10}$

$$
\hat{\tau}=\tau-\frac{1}{V} \sum_{i} y_{i} f_{x, i} .
$$

The second term, sometimes called the "inner virial", stands for a sum over all particles $i$ with $y_{i}$ being the $y$-coordinate of the particle and $f_{x, i}$ the $x$-coordinate of the force acting on the particle. This contribution indeed vanishes on average as can be seen using Eq. (48),

$$
\left\langle y_{i} f_{x, i}\right\rangle=-k_{\mathrm{B}} T\left\langle\frac{\partial y_{i}}{\partial x_{i}}\right\rangle=0,
$$

which shows that $\langle\hat{\tau}\rangle=\tau$ as it must. The stress fluctuations may thus be expressed as

$$
\begin{aligned}
\mu_{\mathrm{F}} & =\beta V\left\langle(\hat{\tau}-\tau)^{2}\right\rangle \\
& =\frac{\beta}{V}\left\langle\sum_{i} y_{i} f_{x, i} \sum_{j} y_{j} f_{x, j}\right\rangle \\
& =-\frac{1}{V} \sum_{i}\left\langle y_{i} \sum_{j} \frac{\partial y_{j} f_{x, j}}{\partial x_{i}}\right\rangle,
\end{aligned}
$$

where we have used the integration by part, Eq. (49). This step requires that all particle positions are unconstrained and independent (generalized) coordinates. Note that this is possible at imposed $\tau$, but cannot hold at fixed $\gamma$. Assuming then pair interactions and using Eq. (40) one can reformulate Eq. (51) within a few lines. Without further approximation this yields

$$
\left.\mu_{\mathrm{F}}\right|_{\tau}=-\frac{1}{V}\left\langle\sum_{l} y_{l}^{2} \frac{\partial f_{x, l}}{\partial x_{l}}\right\rangle,
$$

where the sum runs now over all interactions $l$ between particles $i<j . x_{l}$ and $y_{l}$ refer to components of the distance vector of the interaction $l$ and $f_{x, l}$ to the $x$-component of the central force $f\left(r_{l}\right)=-u^{\prime}\left(r_{l}\right)$ between a particle pair at a distance $r_{l}$. Note that in Eq. (52) the stress fluctuations stemming from different interactions are decoupled, i.e., $\left.\mu_{\mathrm{F}}\right|_{\tau}$ characterizes the self- or two-point contributions of directly interacting beads. Since $f_{x}(r)=-u^{\prime}(r) x / r$ and, hence,

$$
\begin{aligned}
-y^{2} \frac{\partial f_{x}(r)}{\partial x}= & \left(r^{2} u^{\prime \prime}(r)-r^{2} u^{\prime}(r)\right) n_{x}^{2} n_{y}^{2} \\
& -r u^{\prime}(r) n_{x} n_{y},
\end{aligned}
$$

this is identical to the affine shear elasticity $\mu_{\mathrm{A}}$ derived at the end of Sec. II B. We have thus confirmed Eq. (19). Interestingly, the argument can be turned around:

(1) The stress fluctuation contribution term $\left.\mu_{\mathrm{F}}\right|_{\tau}=\mu_{\mathrm{A}}$ is obtained by the simple derivation given in this paragraph.

(2) Using the general Legendre transformation, Eq. (18), this implies $G_{\tau \tau}=\mu_{\mathrm{A}}-\mu_{\mathrm{F}}$ for the $\mathrm{NV} \gamma \mathrm{T}$ - and the $\mathrm{NP} \gamma \mathrm{T}$-ensemble.

As already stressed, our thermodynamic derivation of Eq. (3) is rather general, not using in particular a well-defined displacement field for the particle positions.

\section{Impulsive corrections for truncated potentials}

It is common practice in computational physics ${ }^{7,10}$ to truncate a pair interaction potential $u(r)$, with $r$ being the distance between two particles $i$ and $j$, at a convenient cutoff $r_{\mathrm{c}}$. While this truncation allows to reduce the number of interactions to be computed, it introduces technical difficulties, e.g., instabilities in the numerical solution of differential equations 
as investigated especially for the MD method. ${ }^{10}$ Without restricting much the generality of our results, we assume below that

- the potential scales as $u\left(r, \sigma_{l}\right) \equiv u(s)$ with the reduced dimensionless distance $s=r / \sigma_{l}$ where $\sigma_{l}$ characterizes the length scale of the interaction $l$ and

- the same reduced cutoff $s_{\mathrm{c}}=r_{\mathrm{c}} / \sigma_{l}$ is set for all interactions $l$.

For monodisperse particles with constant diameter $\sigma$, as for the LJ potential $u_{\mathrm{LJ}}(s)=4 \epsilon\left(s^{-12}-s^{-6}\right)$, the scaling variable becomes $s=r / \sigma$ and the reduced cutoff $s_{\mathrm{c}}=r_{\mathrm{c}} / \sigma$. The effect of introducing $s_{\mathrm{c}}$ is to replace $u(s)$ by the truncated potential

$$
u_{\mathrm{t}}(s)=u(s) H\left(s_{\mathrm{c}}-s\right)
$$

with $H(s)$ being the Heaviside function. ${ }^{46}$ Even if Eq. (54) is taken by definition as the system Hamiltonian, impulsive corrections at the cutoff have to be taken into account for the excess pressure $P_{\mathrm{ex}}$ and other moments of the first derivatives of the potential ${ }^{7}$ as may be seen considering the derivative of the truncated potential

$$
u_{\mathrm{t}}^{\prime}(s)=u^{\prime}(s) H\left(s_{\mathrm{c}}-s\right)-u(s) \delta\left(s-s_{\mathrm{c}}\right) .
$$

These corrections with respect to first derivatives can be avoided by considering a properly shifted potential ${ }^{7}$

$$
u_{\mathrm{s}}(s)=\left(u(s)-u\left(s_{\mathrm{c}}\right)\right) H\left(s_{\mathrm{c}}-s\right)
$$

since $u_{\mathrm{s}}^{\prime}(s)=u^{\prime}(s) H\left(s_{\mathrm{c}}-s\right)$. With this choice no impulsive corrections arise for moments of the instantaneous shear stress $\hat{\tau}$ and of other components of the excess stress tensor $\hat{\sigma}_{\alpha \beta}^{\mathrm{ex}}$, Eq. (41). Specifically, if the potential is shifted, all impulsive corrections are avoided for the shear stress fluctuations $\mu_{\mathrm{F}}$, Eq. (2).

The standard shifting of a truncated potential is, however, insufficient in general for properties involving higher derivatives of the potential. ${ }^{42}$ This is the case for the Born coefficient $\mu_{\mathrm{B}}$, Eq. (45), which is required to compute the affine shear elasticity $\mu_{\mathrm{A}}$, Eq. (4). The second derivative of the truncated and shifted potential being

$$
u_{\mathrm{s}}^{\prime \prime}(s)=u^{\prime \prime}(s) H\left(s_{\mathrm{c}}-s\right)-u^{\prime}(s) \delta\left(s-s_{\mathrm{c}}\right),
$$

this implies that the Born coefficient reads $\mu_{\mathrm{B}}=\tilde{\mu}_{\mathrm{B}}-\Delta \mu_{\mathrm{B}}$ with $\tilde{\mu}_{\mathrm{B}}$ being the bare Born coefficient and $\Delta \mu_{\mathrm{B}}$ the impulsive correction which needs to be taken into account. The latter correction may be readily computed numerically from the configuration ensemble using ${ }^{42}$

$$
\Delta \mu_{\mathrm{B}}=\lim _{s \rightarrow s_{\mathrm{c}}^{-}} h_{\mathrm{B}}(s)
$$

with

$$
h_{\mathrm{B}}(s) \equiv \frac{1}{d(d+2) V} \sum_{l}\left\langle s_{l}^{2} u^{\prime}\left(s_{l}\right) \delta\left(s_{l}-s\right)\right\rangle
$$

being a pair distribution function which is related to the standard radial pair distribution function $g(r) .{ }^{48}$ Since $G_{\tau \tau}=\mu_{\mathrm{B}}$ $-P_{\mathrm{ex}}-\mu_{\mathrm{F}}$, Eq. (58) implies in turn

$$
G_{\tau \tau}=\tilde{G}_{\tau \tau}-\Delta \mu_{\mathrm{B}}
$$

with $\tilde{G}_{\tau \tau}$ being the uncorrected bare shear modulus. As we shall see in Sec. IV A, the correction $\Delta \mu_{\mathrm{B}}$ is of importance for the precise determination of $G_{\tau \tau}$ close to the glass transition where the shear modulus must vanish. An impulsive correction has also to be taken into account for the compression modulus $K=K_{p p}$ computed from the normal pressure fluctuations in a constant volume ensemble as discussed in the Appendix. Using the symmetry of isotropic systems, Eq. (A24), one verifies ${ }^{42}$

$$
K_{p p}=\tilde{K}_{p p}-\frac{2+d}{d} \Delta \mu_{\mathrm{B}}
$$

with $\tilde{K}_{p p}$ being the uncorrected compression modulus.

We consider below mixtures and polydisperse systems where $\sigma_{l}$ may differ for each interaction $l$. For such potentials $u(s), u_{\mathrm{t}}(s)$, and $u_{\mathrm{s}}(s)$ and their derivatives take in principal an explicit index $l$, i.e., one should write $u_{l}(s), u_{\mathrm{t}, l}(s), u_{\mathrm{s}, l}(s)$, and so on. For example one might wish to consider the generalization of the monodisperse LJ potential to a mixture or polydisperse system with

$$
u_{l}(s)=4 \epsilon_{l}\left(s^{-12}-s^{-6}\right) \text { with } s=r / \sigma_{l},
$$

where $\epsilon_{l}$ and $\sigma_{l}$ are fixed for each interaction $l$. In practice, each particle $i$ may be characterized by an energy scale $E_{i}$ and a "diameter" $D_{i}$. The interaction parameters $\epsilon_{l}\left(E_{i}, E_{j}\right)$ and $\sigma_{l}\left(D_{i}, D_{j}\right)$ are then given in terms of specified functions of these properties. ${ }^{15}$ The extensively studied Kob-Andersen (KA) model for binary mixtures of beads of type $\mathrm{A}$ and $\mathrm{B},{ }^{49}$ is a particular case of Eq. (61) with fixed interaction ranges $\sigma_{\mathrm{AA}}, \sigma_{\mathrm{BB}}$, and $\sigma_{\mathrm{AB}}$ and energy parameters $\epsilon_{\mathrm{AA}}, \epsilon_{\mathrm{BB}}$, and $\epsilon_{\mathrm{AB}}$ characterizing, respectively, $\mathrm{AA}-, \mathrm{BB}-$, and $\mathrm{AB}$-contacts. The expressions Eqs. (58), (59), and (60) remain valid for such potentials. $^{42}$

\section{ALGORITHMIC AND TECHNICAL ISSUES}

The computational data presented in this work have been obtained using two well-known models of colloidal glassformers. ${ }^{14,15,19,49}$ We present first both models and the MD and $\mathrm{MC}^{7,10,27}$ methods used to compute them in different ensembles. We comment then on the quench protocol. The glass transition temperature $T_{\mathrm{g}}$ for both models is located via dilatometry, as shown in Fig. 2. This is needed as a prerequisite to our study of the elastic behavior in Sec. IV. As sketched in Fig. 1, periodic boundary conditions are applied in all spatial directions with $L_{x}=L_{y}=\cdots$ for the linear dimensions of the $d$-dimensional simulation box. Time scales are measured in units of the $\mathrm{LJ}$ time $\tau_{\mathrm{LJ}}=\left(m \sigma^{2} / \epsilon\right)^{1 / 210}$ for our MD simulations ( $m$ being the particle mass, $\sigma$ the reference length scale, and $\epsilon$ the LJ energy scale) and in units of Monte Carlo Steps (MCS) for our MC simulations. LJ units are used throughout this work $(\epsilon=\sigma=m=1)$ and Boltzmann's constant $k_{\mathrm{B}}$ is also set to unity.

The KA model ${ }^{49}$ for binary mixtures of LJ beads in $d=3$ dimensions has been investigated by means of MD simulation ${ }^{7}$ taking advantage of the public domain LAMMPS implementation. ${ }^{35}$ We use $N=n_{\mathrm{A}}+n_{\mathrm{B}}=6912$ beads per simulation box and molar fractions $c_{a}=n_{\mathrm{A}} / n=0.8$ and $c_{b}$ $=n_{\mathrm{B}} / n=0.2$ for both types of beads $A$ and $B$. Following 


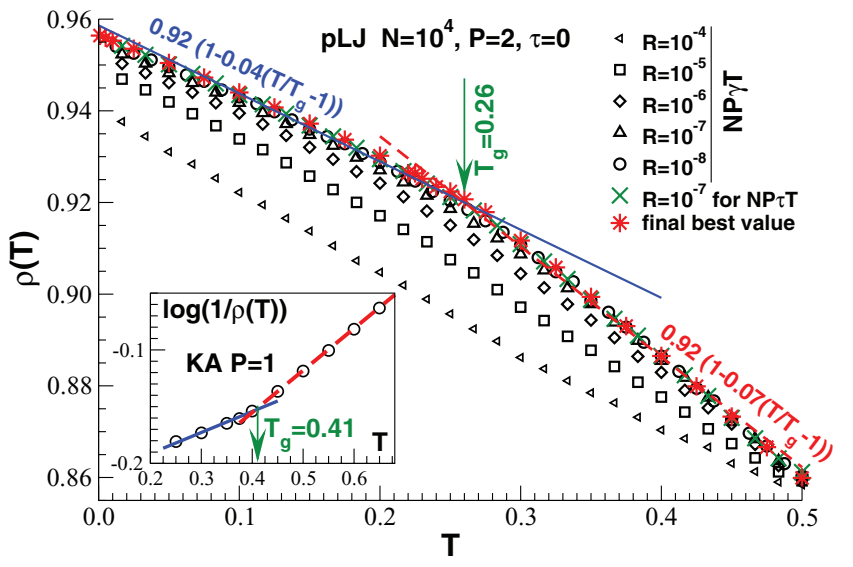

FIG. 2. Density $\rho(T)$ and determination of glass transition temperature $T_{\mathrm{g}}$. Inset: Plotting $\log (1 / \rho)$ vs. $T$ for the KA model as suggested in Ref. 8 reveals a linear low- $T$ (solid line) and a linear high- $T$ regime which confirms $T_{\mathrm{g}} \approx 0.41 .{ }^{49}$ Main panel: Number density $\rho$ for $\mathrm{pLJ}$ systems at constant normal pressure $P=2$ as a function of temperature $T$ for different quench rates $R$ in units of MCS. The crosses refer to a quench in the NP $\tau$ T-ensemble where the box shape is allowed to fluctuate at $\tau=0$. All other data refer to the $\mathrm{NP} \gamma \mathrm{T}$-ensemble with $\gamma=0$. The solid line and the dashed line are linear fits to the best density estimates (stars) obtained for, respectively, low and high temperatures. By matching both lines one determines $T_{\mathrm{g}} \approx 0.26$. A similar value is obtained if $\log (1 / \rho)$ is plotted as a function of $T$.

Ref. 49 we set $\sigma_{\mathrm{AA}}=1.0 \sigma, \sigma_{\mathrm{BB}}=0.88 \sigma$, and $\sigma_{\mathrm{AB}}=0.8 \sigma$ for the interaction range and $\epsilon_{\mathrm{AA}}=1.0 \epsilon, \epsilon_{\mathrm{BB}}=0.5 \epsilon$, and $\epsilon_{\mathrm{AB}}$ $=1.5 \epsilon$ for the LJ energy scales. Only data for the usual (reduced) cutoff $s_{\mathrm{c}}=r_{\mathrm{c}} / \sigma_{l}=2.5$ are presented. For the NP $\gamma \mathrm{T}$ ensemble we use the Nosé-Hoover barostat provided by the LAMMPS code ("fix npt command") 35 which is used to impose a constant pressure $P=1$ for all temperatures. Starting with an equilibrated configuration at $T_{\mathrm{i}}=0.7$ well above the glass transition temperature $T_{\mathrm{g}}=0.41$ (as confirmed in the inset of Fig. 2), the system is slowly quenched in the NP $\gamma \mathrm{T}$ ensemble. The imposed mean temperature $T(t)$ varies linearly as $T(t)=T_{\mathrm{i}}-R t$ with $R=5 \times 10^{-5}$ being the constant quench rate. After a tempering time $t_{\mathrm{eq}}=10^{4}$ we compute over a sampling time $t=5 \times 10^{4}$ various properties for the isobaric system at fixed temperature such as the moduli $G_{\tau \tau}$ represented by the filled spheres in Fig. 10. Fixing only then the volume $(\mathrm{NV} \gamma \mathrm{T}$-ensemble) the system is again tempered $\left(t_{\mathrm{eq}}=10^{4}\right)$ and various properties as the ones recorded in Table I are computed using a sampling time $t=5 \times 10^{4}$. Note that in the $\mathrm{NV} \gamma \mathrm{T}$-ensemble the equations of motion are integrated using a velocity Verlet algorithm ${ }^{10}$ with a time step $\delta t=0.01$ and systems are kept at the imposed temperature $T$ using a Langevin thermostat ${ }^{10}$ with a friction coefficient 0.5 . By redoing for several temperatures around and below $T_{\mathrm{g}}$ the tempering and the production runs we have checked that the presented results remain unchanged (within numerical accuracy) and that ageing effects are negligible. Averages are taken over four independent configurations. As one may expect from the discussion in Sec. II C, very similar results have been found for both ensembles for simple averages, for instance for the affine shear elasticity $\mu_{\mathrm{A}}$, and for the shear modulus $G_{\tau \tau}$, Eq. (3). Unfortunately, we are yet unable to present data for the KA model data at imposed shear stress $\tau$ (NV $\tau \mathrm{T}$-, $\mathrm{NP} \tau \mathrm{T}$-ensembles).

A systematic comparison of constant- $\gamma$ and constant- $\tau$ ensembles has been performed, however, by MC simulation of a specific case of the generalized LJ potential, Eq. (61), where all interaction energies are identical, $\epsilon_{l}=\epsilon$, and the interaction range is set by the Lorentz rule $\sigma_{l}=\left(D_{i}+D_{j}\right) / 2^{48}$ with $D_{i}$ and $D_{j}$ being the diameters of the interacting particles. Only the strictly two-dimensional (2D) case $(d=2)$ is considered. Following Ref. 15, the bead diameters of this polydisperse $\mathrm{LJ}(\mathrm{pLJ})$ model are uniformly distributed between $0.8 \sigma$ and $1.2 \sigma$. For the examples reported in Sec. IV we have used $N=10000$ beads per box. We only present data for a reduced cutoff $s_{\mathrm{c}}=2.0 s_{0}$ with $s_{0}=2^{1 / 6}$ being the the minimum of the polydisperse LJ potential, Eq. (61). Various properties obtained for the $\mathrm{pLJ}$ model are summarized in Table II.

For all ensembles considered here local MC moves are used (albeit not exclusively) where a particle is chosen randomly and a displacement $\delta \underline{r}$, uniformly distributed over a disk of radius $\delta r_{\max }$, from the current position $\underline{r}$ of the particle is attempted. The corresponding energy change $\delta E$ is calculated and the move is accepted using the standard Metropolis criterion. ${ }^{27}$ The maximum displacement distance $\delta r_{\max }$ is chosen such that the acceptance rate $A$ remains reasonable, i.e., $0.1 \leq A \leq 0.5 .{ }^{27}$ As may be seen from the inset in Fig. 3 where the acceptance rate $A(T)$ is shown as a function

TABLE I. Some properties for the KA model at normal pressure $P=1$ and shear stress $\tau=0$ : the mean density $\rho$, the mean energy per volume $e \rho$, the impulsive correction term $\Delta \mu_{\mathrm{B}}$ (Sec. II D), the (corrected) Born-Lamé coefficient $\mu_{\mathrm{B}}$, the affine shear elasticity $\mu_{\mathrm{A}}=\mu_{\mathrm{B}}-P_{\mathrm{ex}}$, the shear modulus $G_{\tau \tau}$ obtained in the $\mathrm{NV} \gamma \mathrm{T}$-ensemble, the hypervirial $\eta_{\mathrm{B}}$ and the affine dilatation elasticity $\eta_{\mathrm{A}}=2 P_{\text {id }}+\eta_{\mathrm{B}}+P_{\text {ex }}$ discussed in the Appendix and the compression modulus $K_{p p}$ obtained for the $\mathrm{NV} \gamma \mathrm{T}$-ensemble. The affine elasticities $\mu_{\mathrm{A}}$ and $\eta_{\mathrm{A}}$ are the natural scales for, respectively, the shear modulus $G$ and the compression modulus $K$.

\begin{tabular}{|c|c|c|c|c|c|c|c|c|c|}
\hline$T$ & $\rho$ & $e \rho$ & $\Delta \mu_{\mathrm{B}}$ & $\mu_{\mathrm{B}}$ & $\mu_{\mathrm{A}}$ & $G_{\tau \tau}$ & $\eta_{\mathrm{B}}$ & $\eta_{\mathrm{A}}$ & $K_{p p}$ \\
\hline 0.025 & 1.24 & -9.42 & 0.80 & 52.1 & 51.15 & 21.9 & 86.2 & 87.2 & 82.4 \\
\hline 0.100 & 1.20 & -8.85 & 0.80 & 50.4 & 49.51 & 19.6 & 83.4 & 84.5 & 75.7 \\
\hline 0.200 & 1.21 & -8.57 & 0.78 & 48.3 & 47.52 & 17.3 & 80.0 & 81.2 & 67.7 \\
\hline 0.250 & 1.20 & -8.31 & 0.77 & 47.2 & 46.54 & 13.9 & 78.2 & 79.6 & 63.1 \\
\hline 0.310 & 1.19 & -7.99 & 0.74 & 45.7 & 45.10 & 11.8 & 75.8 & 77.2 & 56.9 \\
\hline 0.350 & 1.18 & -7.82 & 0.73 & 45.1 & 44.45 & 9.3 & 73.9 & 76.1 & 52.6 \\
\hline 0.375 & 1.17 & -7.67 & 0.72 & 44.6 & 43.96 & 6.1 & 73.9 & 75.3 & 50.5 \\
\hline 0.400 & 1.17 & -7.49 & 0.71 & 43.8 & 43.24 & 2.5 & 72.6 & 74.1 & 45.3 \\
\hline 0.450 & 1.15 & -7.07 & 0.68 & 42.0 & 41.48 & 0.03 & 69.6 & 71.1 & 37.9 \\
\hline 0.800 & 1.00 & -4.49 & 0.55 & 29.7 & 29.5 & $\approx 0$ & 49.4 & 51.2 & 16.6 \\
\hline
\end{tabular}


TABLE II. Some properties of the pLJ model at $P=2$ and $\tau=0$ : the mean density $\rho$, the mean energy per volume $e \rho$, the impulsive correction term $\Delta \mu_{\mathrm{B}}$, the affine shear elasticity $\mu_{\mathrm{A}}$, the shear moduli $G_{\gamma \tau}$ and $G_{\gamma \gamma}$ obtained in the NP $\tau$ T-ensemble and $G_{\tau \tau}$ obtained in the NV $\gamma \mathrm{T}$-ensemble, the affine dilatation elasticity $\eta_{\mathrm{A}}$, the compression modulus $K_{v p}$ obtained in the NP $\tau$ T-ensemble, and the Rowlinson formula $K_{p p}$ for the NV $\gamma \mathrm{T}$-ensemble. All data have been obtained for a sampling time $t=10^{7}$ MCS.

\begin{tabular}{lccccccccc}
\hline \hline$T$ & $\rho$ & $e \rho$ & $\Delta \mu_{\mathrm{B}}$ & $\mu_{\mathrm{A}}$ & $G_{\gamma \tau}$ & $G_{\gamma \gamma}$ & $G_{\tau \tau}$ & $\eta_{\mathrm{A}}$ & $K_{v p}$ \\
\hline 0.001 & 0.96 & -2.70 & 0.31 & 33.9 & 15.6 & 15.7 & 15.5 & 71.7 & 70.8 \\
0.010 & 0.96 & -2.69 & 0.38 & 33.7 & 14.3 & 14.2 & 13.0 & 71.3 & 69.7 \\
0.100 & 0.94 & -2.60 & 0.39 & 32.4 & 10.9 & 10.9 & 10.3 & 68.8 & 61.2 \\
0.200 & 0.93 & -2.48 & 0.38 & 31.1 & 7.2 & 7.2 & 7.4 & 66.2 & 51.2 \\
0.225 & 0.93 & -2.45 & 0.38 & 30.6 & 4.6 & 4.6 & 3.7 & 65.3 & 47.9 \\
0.250 & 0.92 & -2.42 & 0.37 & 30.0 & 1.4 & 1.4 & 0.94 & 64.0 & 41.0 \\
0.275 & 0.92 & -2.38 & 0.37 & 29.7 & 0.6 & 0.6 & 0.05 & 63.4 & 40.0 \\
0.300 & 0.91 & -2.33 & 0.35 & 29.3 & 0.1 & 0.1 & 0.09 & 62.6 & 37.3 \\
0.325 & 0.91 & -2.29 & 0.39 & 28.9 & $\approx 0$ & $\approx 0$ & $\approx 0$ & 61.7 & 34.0 \\
0.350 & 0.90 & -2.24 & 0.33 & 28.3 & $\approx 0$ & $\approx 0$ & $\approx 0$ & 60.5 & 37.4 \\
1.000 & 0.73 & -1.23 & 0.22 & 17.0 & $\approx 0$ & $\approx 0$ & $\approx 0$ & 38.0 & 9.3 \\
\hline \hline
\end{tabular}

of temperature $T$, we find that temperatures below $T=0.1$ are best sampled using $\delta r_{\max } \approx 0.01$, whereas $\delta r_{\max } \approx 0.1$ is a good choice for the interesting temperature regime between $T=0.2$ and $T=0.3$ around the glass transition temperature $T_{\mathrm{g}}=0.26$. See the main panel of Fig. 2 for the determination of $T_{\mathrm{g}}$. Various data sets are presented in Sec. IV for a fixed value $\delta r_{\max }=0.1$ which allows a reasonable comparison of the sampling time dependence for temperatures around the glass transition. This value is not necessarily the best choice for tempering the system and for computing static equilibrium properties at the given temperature, especially below $T \approx 0.2$.

Local MC jumps in the NV $\gamma$ T-ensemble become obviously inefficient for relaxing large scale structural properties ${ }^{27}$ for the lowest temperatures computed for the $\mathrm{pLJ}$ model $(T \leq 0.1)$. We have thus crosschecked and improved the values obtained using only local MC moves by adding global collective MC move attempts for all particles with longitudinal and (more importantly) transverse plane waves com-

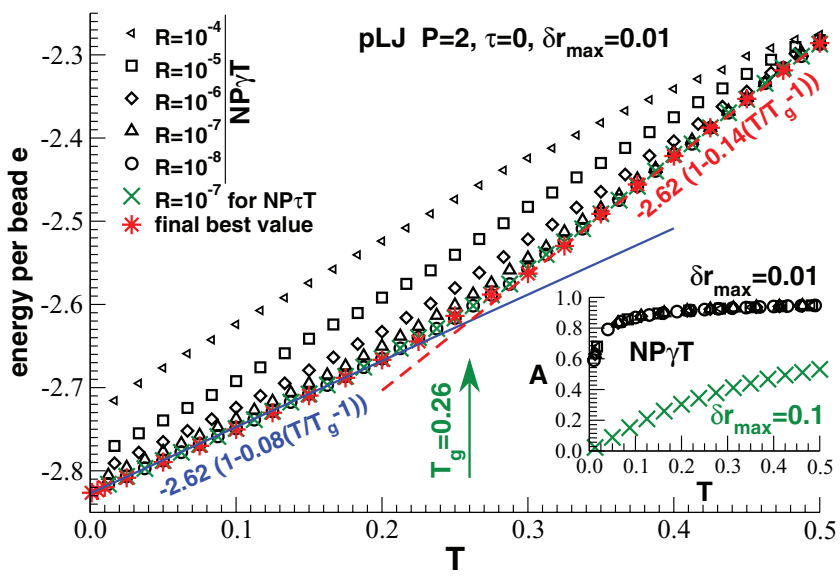

FIG. 3. Energy per particle $e$ (main panel) and acceptance rate $A$ (inset) obtained for $\mathrm{pLJ}$ systems quenched at constant pressure $P=2$ at a given quench rate $R$ as indicated. The crosses refer to a quench at imposed $\tau=0$. The solid line and the dashed line are linear fits to the energy for, respectively, low and high temperatures confirming the value $T_{\mathrm{g}} \approx 0.26$ for the $\mathrm{pLJ}$ model. Only local MC moves with one fixed (non-adaptive) maximum particle displacement $\delta r_{\max }$ for all temperatures $T$ have been used for the MC data shown in the main panel. The acceptance rate decreases thus with decreasing $T$ as shown in the inset for $\delta r_{\max }=0.01$ (upper data) and $\delta r_{\max }=0.1$. mensurate with the simulation box. The random amplitudes and phases of the waves are assumed to be uniformly distributed. Note that the maximum amplitude $a_{\max }(q)$ of each wave is chosen inversely proportional to the length $q=|q|$ of the wavevector in agreement with continuum theory. This allows to keep the same acceptance rate for each global mode if $q \ll 1$. As one expects, the plane-wave displacement attempts becomes inappropriate for large $q$ where continuum elasticity breaks down ${ }^{14,15,19}$ and, hence, the acceptance rate $A(q)$ gets too large to be efficient. These global plane-wave moves have been added for the tempering of all presented configurations below $T=0.3$ with $t_{\mathrm{eq}}=10^{7}$ and for production runs over sampling times $t=10^{7}$ for static properties for $T<0.2$.

Using again the Metropolis criterion for accepting a suggested change of the overall box volume $V$ or of the shear strain $\gamma$, pure dilatational and pure shear strain fluctuations are used to impose a mean normal pressure $P=2$ and a mean shear stress $\tau=0$. For a shear strain altering MC move one first chooses randomly a strain fluctuation $\delta \gamma$ with $|\delta \gamma|$ $\leq \delta \gamma_{\max }$. Assuming an imposed shear stress $\tau$ the suggested $\delta \gamma$ is accepted if

$$
\xi \leq \exp (-\beta \delta E+\beta \delta \gamma \tau)
$$

with $\xi$ being a uniformly distributed random variable with $0 \leq \xi<1$. The energy change $\delta E$ associated with the affine strain may be calculated using Eq. (33). Since $\tau=0$ is supposed in this study, the last term in the exponential drops out. The maximum shear strain step $\delta \gamma_{\max }$ is fixed such that at the given temperature the acceptance rate remains reasonable. ${ }^{50}$ As described in Ref. 10, one similarly chooses a relative volume change $\delta V / V$ with $V$ being the current volume. The volume altering move is accepted at an imposed pressure $P$ if

$$
\xi \leq \exp (-\beta \delta E+N \log (1+\delta V / V)-\beta \delta V P),
$$

where $\delta E$ may be computed using Eq. (A16). The logarithmic contribution corresponds to the change of the translational entropy. Using Eq. (62) after each MCS for local MC moves realizes the NV $\tau$ T-ensemble shown in Fig. 1(c), using Eq. (63) the NP $\gamma$ T-ensemble shown in Fig. 1(d) and using in turn both strain fluctuations the NP $\tau$ T-ensemble in Fig. 1(e). We remind that $\mathrm{NV} \gamma \mathrm{T}$ - and $\mathrm{NP} \gamma \mathrm{T}$-ensembles are 
used to determine $G_{\tau \tau}$ and $\mathrm{NV} \tau \mathrm{T}$ - and $\mathrm{NP} \tau \mathrm{T}$-ensembles to obtain $G_{\gamma \tau}$ and $G_{\gamma \gamma}$. As shown in Fig. 2, we quench the configurations starting from $T_{\mathrm{i}}=0.5$ in the NP $\gamma \mathrm{T}$ - and the $\mathrm{NP} \tau \mathrm{T}$-ensemble using a constant quench rate $R$. Note that the smallest quench rate $R=10^{-8}$ for one configuration in the NP $\gamma \mathrm{T}$-shown did require alone a run over six months using one core of an Intel Xeon E5410 processor. Interestingly, similar results are obtained with the $\mathrm{NP} \tau \mathrm{T}$-ensemble only using a rate $R=10^{-7}$. The configurations created by the $\mathrm{NP} \gamma \mathrm{T}$-quenches are used (after tempering) for the sampling in the $\mathrm{NV} \gamma \mathrm{T}$ - and $\mathrm{NP} \gamma \mathrm{T}$-ensembles, the configurations obtained by the $\mathrm{NP} \tau \mathrm{T}$-quenches for the sampling in the NV $\tau \mathrm{T}$ and $\mathrm{NP} \tau \mathrm{T}$-ensembles. Only two independent configurations have been sampled following the full protocol for each temperature and each of the four ensembles. Instead of increasing further the number of configurations we have focused in the present study on long tempering times and production runs over $t=10^{7}$ MCS which have been redone several times for a few temperatures to check for equilibration problems and to verify that ageing effects can be ignored for the properties considered in this work.

\section{COMPUTATIONAL RESULTS}

\section{A. High-temperature liquid limit}

We begin our discussion by focusing on the high temperature liquid limit where all reasonable operational definitions of the shear modulus $G$ must vanish. The results presented in Fig. 4 have been obtained for the pLJ model at mean pressure $P=2$, mean shear stress $\tau=0$ and mean temperature $T=1$, i.e., far above the glass transition temperature $T_{\mathrm{g}} \approx 0.26$. Only local $\mathrm{MC}$ monomer displacements with a maximum jump displacement $\delta r_{\max }=0.1$ are reported here. Instantaneous properties relevant for the moments are writ-

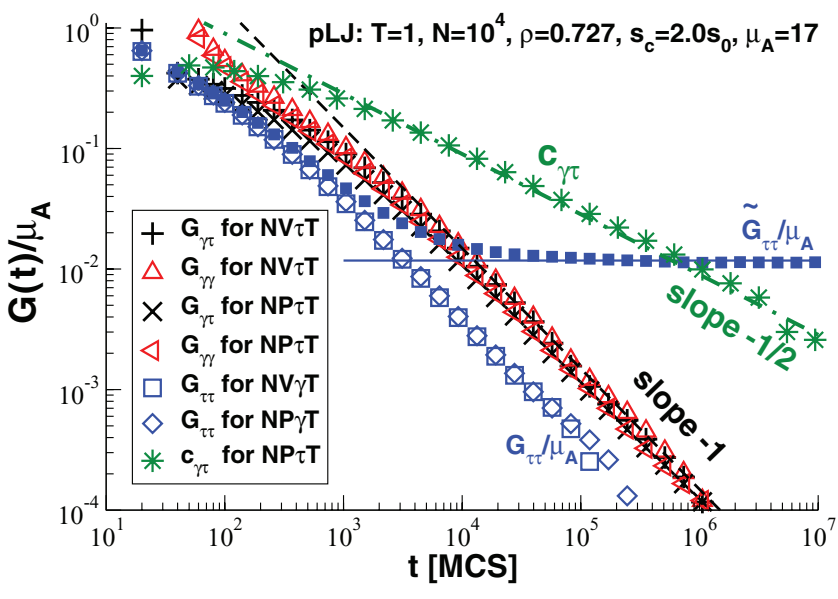

FIG. 4. Rescaled shear modulus $G(t) / \mu_{\mathrm{A}}$ vs. sampling time $t$ in the high$T$ liquid limit of the pLJ model comparing various ensembles and observables. The filled squares show the bare shear modulus $\tilde{G}_{\tau \tau}$ obtained using Eq. (3) for the $\mathrm{NV} \gamma \mathrm{T}$-ensemble without the impulsive correction $\Delta \mu_{\mathrm{B}} \approx 0.2$ (horizontal line). The corrected modulus $G_{\tau \tau}=\tilde{G}_{\tau \tau}-\Delta \mu_{\mathrm{B}}$ for the NV $\gamma \mathrm{T}$ ensemble (open squares) and the NP $\gamma \mathrm{T}$-ensemble (open diamonds) vanishes as it should. Note that $G_{\tau \tau}$ decreases slightly faster than $G_{\gamma \tau} \approx G_{\gamma \gamma}$. As shown by the dashed line, all operational definitions of $G$ decay inversely with $t$. The dashed-dotted line indicates the expected power-law exponent $-1 / 2$ for the correlation coefficient $c_{\gamma \tau}$ (stars). ten down every 10 MCS and averaged using standard gliding averages,${ }^{10}$ i.e., we compute mean values and fluctuations for a given time interval $\left[t_{0}, t_{1}=t_{0}+t\right]$ and average over all possible intervals of length $t$. The horizontal axis indicates the latter interval length $t$ in units of MCS. The vertical axis is made dimensionless by rescaling the moduli with the (corrected) affine shear elasticity $\mu_{\mathrm{A}}=\mu_{\mathrm{B}}-P_{\mathrm{ex}} \approx 17$ from Table II. (Being a simple average, $\mu_{\mathrm{A}}$ is found to be identical for all computed ensembles.)

In agreement with Eq. (21), the ratio $G(t) / \mu_{\mathrm{A}}$ for all operational definitions drops rapidly below unity. The dashed line indicates the asymptotic power-law slope $-1{ }^{22,42}$ This can be understood by noting that at imposed $\tau$ the shear strain freely diffuses in the liquid limit, i.e., $\left\langle\delta \hat{\gamma}^{2}\right\rangle \sim t$, which implies that $G \approx G_{\gamma \tau} \sim 1 / t$. For both $\mathrm{NV} \tau \mathrm{T}$ - and $\mathrm{NP} \tau \mathrm{T}$-ensembles we compare our key definition $G_{\gamma \tau}$, Eq. (6), to the observable $G_{\gamma \gamma}$, Eq. (9). In both cases we confirm $G_{\gamma \tau}(t) \approx G_{\gamma \gamma}(t)$ for larger times as suggested by the thermodynamic relation Eq. (8) which has been directly checked..$^{41}$ We have also computed the dimensionless correlation coefficient $c_{\gamma \tau}(t)$, Eq. (7), which characterize the correlation of the measured instantaneous shear strains $\hat{\gamma}$ and shear stresses $\hat{\tau}$. As indicated for the NP $\tau$ T-ensemble (stars), $c_{\gamma \tau}$ vanishes rapidly with $t$, i.e., $\hat{\gamma}$ and $\hat{\tau}$ are decorrelated. The dashed-dotted line indicates the power-law exponent $-1 / 2$ expected from Eq. (20) and $G_{\gamma \tau}(t)$ $\sim 1 / t$. Note that by plotting $c_{\gamma \tau}(t)$ vs. $G_{\gamma \tau}(t) / \mu_{\mathrm{A}}$, Eq. (20) can be directly demonstrated (not shown).

The stress fluctuation formula, Eq. (3), is represented in Fig. 4 by squares and diamonds for, respectively, $\mathrm{NV} \gamma \mathrm{T}$ - and NP $\gamma$ T-ensembles. The bare shear modulus $\tilde{G}_{\tau \tau}$ without the impulsive correction $\Delta \mu_{\mathrm{B}}$ for the Born coefficient $\mu_{\mathrm{B}}$ discussed in Sec. II D is given by small filled symbols. The impulsive correction $\Delta \mu_{\mathrm{B}} \approx 0.2$ (Table II) computed independently from Eq. (58) is shown by a horizontal line. The corrected moduli $G_{\tau \tau}=\tilde{G}_{\tau \tau}-\Delta \mu_{\mathrm{B}}$ vanish indeed as expected. See Ref. 42 for a systematic characterization of $\Delta \mu_{\mathrm{B}}$ as a function of the reduced cutoff $s_{\mathrm{c}}$. As also shown there, a truncation correction is also needed for the KA model. The $\Delta \mu_{\mathrm{B}}$ for different temperatures may be found in Table I for the KA model and in Table II for the pLJ model. We assume from now on that the impulsive corrections are properly taken into account without stating this technical point explicitly.

\section{B. Dynamical matrix harmonic network}

We turn now to the opposite low- $T$ limit where we focus on the pLJ model at $T=0.001$. Obviously, such deeply quenched colloidal glasses must behave as amorphous solids with a constant shear modulus for large times by the top curve. Before presenting in Sec. IV C the elastic properties of these glasses, we discuss first conceptually simpler substitute systems formed by permanent spring networks. We do this for illustration purposes since questions related to ergodicity and ageing are by construction irrelevant and the thermodynamical relations reminded in Sec. II should hold rigorously. Assuming ideal harmonic springs the interaction energy reads $E=\frac{1}{2} \sum_{l} K_{l}\left(r_{l}-R_{l}\right)^{2}$ with $K_{l}$ being the spring constant and 


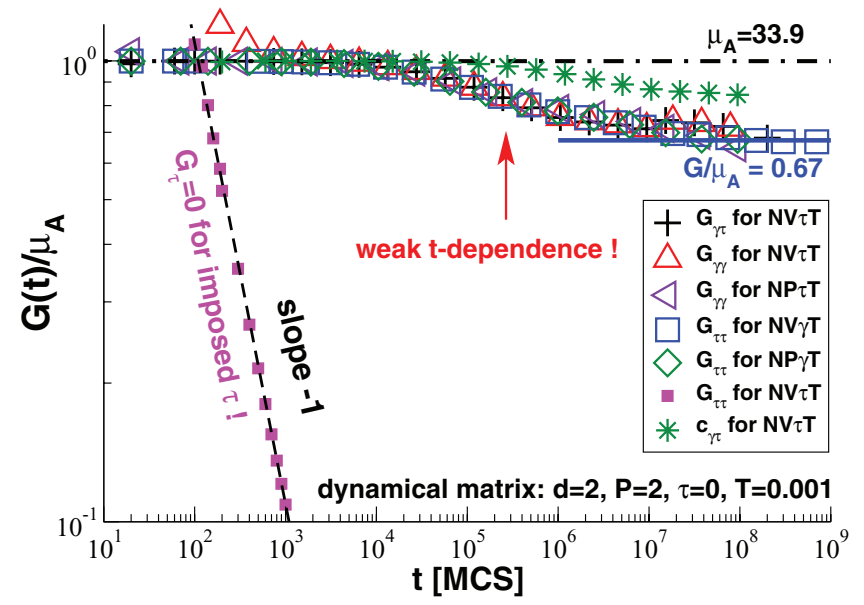

FIG. 5. Rescaled shear modulus $G(t) / \mu_{\mathrm{A}}$ vs. sampling time $t$ for systems of permanent linear springs corresponding to the dynamical matrix of $\mathrm{pLJ}$ systems at $P=2, \tau=0$, and $T=0.001$. As indicated by the bold line, we obtain $G / \mu_{\mathrm{A}} \approx 0.67$ in the long-time limit. In agreement with Eq. (19) $G_{\tau \tau}$ vanishes with time if it is computed in an ensemble where $\gamma$ fluctuates as shown for the NV $\tau$ T-ensemble (filled squares).

$R_{l}$ the reference length of spring $l$. The sum runs over all springs $l$ between topologically connected vertices $i$ and $j$ of the network. We assume here a strongly and homogeneously connected network where every vertex $i$ is in contact with many neighbors $j$. Such a network may be constructed from a pLJ configuration keeping the particle positions for the positions of the vertices and replacing each $\mathrm{LJ}$ interaction $l$ by a spring. Both spring constants are chosen such that the "dynamical matrix" of the pLJ configuration and harmonic network are identical, i.e., the second derivative of the total interaction potential with respect to the particle positions ${ }^{1}$ is the same for $T \rightarrow 0$. This implies

$$
\begin{aligned}
K_{l} & \left.\equiv u^{\prime \prime}(r)\right|_{r=r_{l}}, \\
R_{l} & \equiv r_{l}-\left.\frac{u^{\prime}(r)}{u^{\prime \prime}(r)}\right|_{r=r_{l}}
\end{aligned}
$$

with $u(r)=u(s)$ being the interaction potential for reduced distances $s_{l}=r_{l} / \sigma_{l} \leq s_{\mathrm{c}}$. (Impulsive corrections at $s_{l}=s_{\mathrm{c}}$ are neglected here.) Taking advantage of the fact that all interacting vertices are known, these networks can be computed using global MC moves with longitudinal and transverse planar waves as described in Sec. III. No local MC jumps for individual vertices have been added. Note that although some $K_{l}$ and $R_{l}$ may even be negative, this does not affect the global or local stability of the network. For networks at the same pressure $P$, shear stress $\tau$, and temperature $T$ as the original pLJ configuration, we obtain, not surprisingly, the same affine shear elasticity $\mu_{\mathrm{A}}=33.9$ as for the reference. The same applies for other simple averages.

Figure 5 presents various measurements of the shear modulus $G$ for different ensembles as a function of sampling time $t$. As in Sec. IV A, we use gliding averages and the vertical axis has been rescaled by the affine shear elasticity $\mu_{\mathrm{A}}$. As can be seen, we obtain in the long-time limit $G / \mu_{\mathrm{A}}$ $\approx 0.67$ (bold line) irrespective of whether the shear modulus is determined from $G_{\gamma \tau}$ or $G_{\gamma \gamma}$ in the NV $\tau$ T- or $\mathrm{NP} \tau \mathrm{T}$ -

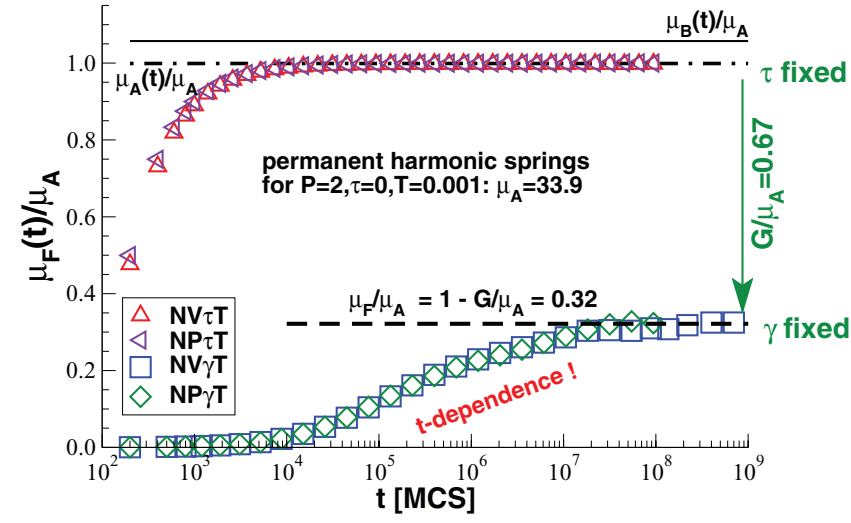

FIG. 6. Shear stress fluctuations $\mu_{\mathrm{F}}(t) / \mu_{\mathrm{A}}$ with $\mu_{\mathrm{A}}=33.9$ for the same systems as in Fig. 5. We observe $\mu_{\mathrm{F}}(t) / \mu_{\mathrm{A}} \rightarrow 1$ (dash-dotted line) for imposed $\tau$, while $\mu_{\mathrm{F}}(t) / \mu_{\mathrm{A}} \rightarrow 1-G / \mu_{\mathrm{A}} \approx 0.32$ (dashed line) for imposed $\gamma$. Note the broad time-dependence of $\mu_{\mathrm{F}}(t)$ in the latter cases.

ensembles or using $G_{\tau \tau}$ in the NV $\gamma \mathrm{T}$ - or NP $\gamma$ T-ensembles at fixed shear strain $\gamma$. Note that even in these cases the shear modulus decreases first with time albeit the network is perfectly at thermodynamic equilibrium and no ageing occurs by construction. As shown by the dash-dotted line, $G(t) / \mu_{\mathrm{A}} \rightarrow 1$ for short times, i.e. in this limit the response is affine. As indicated by the stars, we have also computed the correlation coefficient $c_{\gamma \tau}(t)$ as a function of time. From $c_{\gamma \tau}=1$ for small $t$, it decreases somewhat becoming $c_{\gamma \tau} \approx \sqrt{0.67} \approx 0.82$ for large times in agreement with Eq. (20). If $G_{\tau \tau}$ is computed in the "wrong" NV $\tau$ T- or NP $\tau$ T-ensembles, it is seen to vanish rapidly with time as shown by the filled squares. This decay is of course expected from Eq. (19).

To emphasize the latter point we show in Fig. 6 the stress fluctuations $\mu_{\mathrm{F}}(t)$ for different ensembles. This is also done to have a closer look at the time dependence of various contributions to $G_{\tau \tau}(t)$. As before, the vertical axis has been rescaled with $\mu_{\mathrm{A}} \equiv 33.9$. We also indicate the rescaled values of $\mu_{\mathrm{B}}(t)$ (thin top line) and $\mu_{\mathrm{A}}(t)=\mu_{\mathrm{B}}(t)-P_{\mathrm{ex}}(t)$ (dasheddotted line) computed by a gliding average over time intervals $\left[t_{0}, t_{1}=t_{0}+t\right]$. Being simple averages both properties do not depend on the ensemble probed (not shown) and become virtually immediately $t$-independent as can be seen from the indicated horizontal lines. This is quite different for the different shear stress fluctuations $\mu_{\mathrm{F}}$ presented which increase monotonously from $\mu_{\mathrm{F}} \approx 0$ for short times to their plateau value for long times. In agreement with Eq. (19) we find that $\left.\mu_{\mathrm{F}}(t)\right|_{\tau} \rightarrow \mu_{\mathrm{A}}$ for NV $\tau \mathrm{T}$ - and NP $\tau \mathrm{T}$-ensembles and as predicted by Eq. (18) we confirm $\left.\mu_{\mathrm{F}}(t)\right|_{\gamma} \rightarrow \mu_{\mathrm{A}}-G$ for NV $\gamma \mathrm{T}$ and NP $\gamma$ T-ensembles. The asymptotic limit is reached after about $10^{4}$ MCS in the first case for imposed $\tau$, but only after $10^{7}$ for imposed $\gamma$. This is due to the fact that $\left.\mu_{\mathrm{F}}\right|_{\tau}$ corresponds to the fluctuations of the self-contribution of individual springs as discussed in Sec. II C, while $\left.\mu_{\mathrm{F}}\right|_{\gamma}$ also contains contributions from stress correlations of different springs. It should be rewarding to analyze in the future finite systemsize effects and the time dependence of three-dimensional (3D) systems using the dynamical matrix associated to the KA model. 


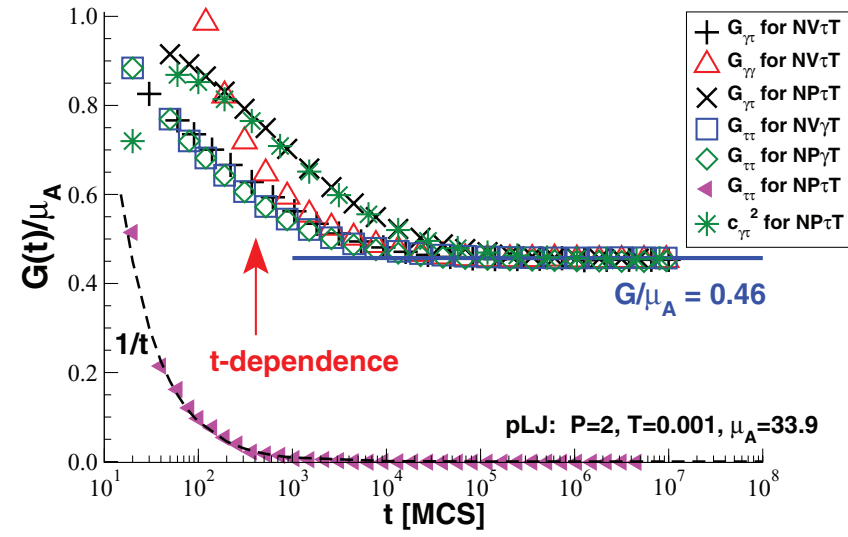

FIG. 7. $G_{\gamma \tau}(t) / \mu_{\mathrm{A}}, G_{\gamma \gamma}(t) / \mu_{\mathrm{A}}$ and $G_{\tau \tau}(t) / \mu_{\mathrm{A}}$ for pLJ beads in the lowtemperature limit for $P=2, \tau=0$ and $T=0.001$ where $\mu_{\mathrm{A}} \approx 33.9$. Qualitatively, the data compares well with the various shear moduli presented in Fig. 5. The filled triangles show $G_{\tau \tau}$ for the NP $\tau$ T-ensemble which is seen to vanish rapidly with sampling time $t$. The dashed line indicates a $1 / t$-decay.

\section{Low-temperature glass limit}

We return now to the colloidal glasses where the topology of the interactions is, of course, not permanently fixed (at least not at finite temperatures) and the shear stresses may thus fluctuate more strongly. As an example, we present in Fig. 7 the shear modulus $G(t)$ as a function of sampling time $t$ for $\mathrm{pLJ}$ beads at mean pressure $P=2$, mean shear stress $\tau=0$ and mean temperature $T=0.001$, i.e., the same conditions as in the previous subsection. All simple averages, such as the affine shear elasticity $\mu_{\mathrm{A}}=33.9$, are the same for all ensembles albeit different quench protocols have been used. For all examples given we use local MC jumps with $\delta r_{\max }=0.01$ which allows to keep a small, but still reasonable acceptance rate $A \approx 0.161$. We have also performed runs with additional global MC moves using longitudinal and transverse plane waves. This yields similar data which is shifted horizontally to the left (not shown).

As one would expect, the shear moduli presented in Fig. 7 are similar to the ones shown in Fig. 5 for the permanent spring network. The filled triangles show $G_{\tau \tau}$ computed in the "wrong" $\mathrm{NP} \tau \mathrm{T}$-ensemble. As expected from Eq. (18) these values vanish rapidly with time. If on the other hand $G_{\gamma \tau}, G_{\gamma \gamma}$, and $G_{\tau \tau}$ are computed in their natural ensembles, all these measures of $G$ are similar and approach, as one expects for such a low temperature, the same finite asymptotic value $G / \mu_{\mathrm{A}} \approx 0.46$ indicated by the bold horizontal line. The stars indicate the squared correlation coefficient $c_{\gamma \tau}^{2}(t)$ which is seen to collapse perfectly on the rescaled modulus $G_{\gamma \tau}(t) / \mu_{\mathrm{A}}$ which shows that Eq. (20) even holds for small times before the thermodynamic plateau has been reached. Similar reduced shear moduli $G_{\tau \tau} / \mu_{\mathrm{A}}$ have been also obtained for the KA model in the low- $T$ limit as may be seen from Table I. These results confirm that about half of the affine shear elasticity is released by the shear stress fluctuations in agreement with Refs. 14 and 15. Interestingly, this value is slightly smaller as the corresponding value for the permanent dynamical matrix model presented above. Since some, albeit very small, rearrangement of the interactions must occur for the $\mathrm{pLJ}$ particles at finite temperature, this is a reasonable finding.

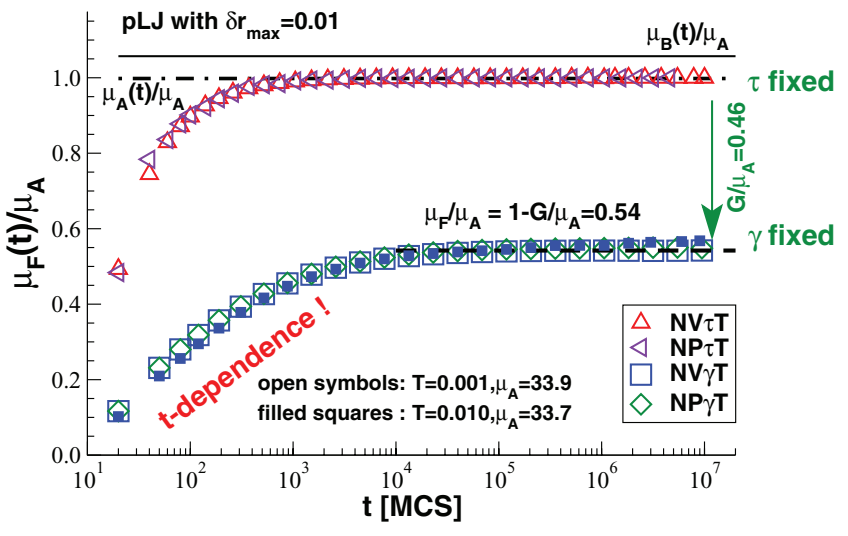

FIG. 8. Reduced shear stress fluctuation $\mu_{\mathrm{F}}(t) / \mu_{\mathrm{A}}$ for $\mathrm{pLJ}$ beads using the same representation as in Fig. 6. The small filled squares refer to data obtained for $T=0.010$ using the NV $\gamma \mathrm{T}$-ensemble, all other data to $T=0.001$. The reduced Born coefficient $\mu_{\mathrm{B}}(t) / \mu_{\mathrm{A}}$ (thin line) and the affine shear elasticity $\mu_{\mathrm{A}}(t) / \mu_{\mathrm{A}}$ (dashed-dotted line) are essentially time independent while $\mu_{\mathrm{F}}(t) / \mu_{\mathrm{A}}$ approaches its large- $t$ limit (dashed line) from below.

The rescaled shear stress fluctuations $\mu_{\mathrm{F}}(t) / \mu_{\mathrm{A}}$ for the same ensembles are presented in Fig. 8. While the two-point correlations $\mu_{\mathrm{B}}(t) / \mu_{\mathrm{A}}$ (thin horizontal line) and $\mu_{\mathrm{A}}(t) / \mu_{\mathrm{A}}$ (dashed-dotted horizontal line) do not dependent on time, one observes again that $\mu_{\mathrm{F}}(t) / \mu_{\mathrm{A}}$ increases monotonously from zero to its long-time asymptotic plateau value. In agreement with Eq. (19) we find $\left.\mu_{\mathrm{F}}(t)\right|_{\tau} \rightarrow \mu_{\mathrm{A}}$ for the $\mathrm{NV} \tau \mathrm{T}$ and the $\mathrm{NP} \tau \mathrm{T}$ ensembles, while $\left.\mu_{\mathrm{F}}(t)\right|_{\gamma} \rightarrow \mu_{\mathrm{A}}-G$ (dashed horizontal line) for the $\mathrm{NV} \gamma \mathrm{T}$ and the NP $\gamma \mathrm{T}$ ensembles. (Similar behavior has been obtained for the KA model in the $\mathrm{NV} \gamma \mathrm{T}$ ensemble for temperatures $T \leq 0.1$.) As may be better seen for the data set obtained for $T=0.010$ using the $\mathrm{NV} \gamma \mathrm{T}$-ensemble (small filled squares) where $\mu_{\mathrm{A}} \approx 33.7$, the stress fluctuations $\left.\mu_{\mathrm{F}}(t)\right|_{\gamma}$ do not rigorously become constant, but increase extremely slowly on the logarithmic time scales presented. There is, hence, even at low temperatures always some sampling time dependence if the interactions are not permanently fixed as for the topologically fixed networks discussed above. However, this effect becomes only sizeable above $T=0.2$ for the KA model and above $T=0.1$ for the pLJ model.

\section{Scaling with temperature}

We turn now to the characterization of the temperature dependence of the shear modulus $G$ and various related properties. We focus first on the best values obtained for the longest simulation runs available. Figure 9 presents the stress fluctuations obtained for the KA model using the $\mathrm{NV} \gamma \mathrm{T}$ ensemble (open spheres) and for the pLJ model using NV $\gamma \mathrm{T}$ and the NP $\tau$ T-ensembles. The affine shear elasticity $\mu_{\mathrm{A}} \mathrm{ob}-$ tained for each system is represented by small filled symbols. As shown for both models by a bold solid line for the low$T$ and by a dashed line for the high- $T$ regime, $\mu_{\mathrm{A}}(T)$ decays roughly linearly with $T$. Interestingly, the slopes match precisely at $T=T_{\mathrm{g}}$ for both models. As may be seen from the large triangles for the $\mathrm{NP} \tau \mathrm{T}$-ensemble, we confirm for all temperatures that $\left.\mu_{\mathrm{F}}\right|_{\tau} \approx \mu_{\mathrm{A}}$ as expected from Eq. (19). For systems without box shape fluctuations, it is seen that $\left.\mu_{\mathrm{F}}\right|_{\gamma}$ becomes non-monotonous with a clear maximum at $T \approx T_{\mathrm{g}}$. 


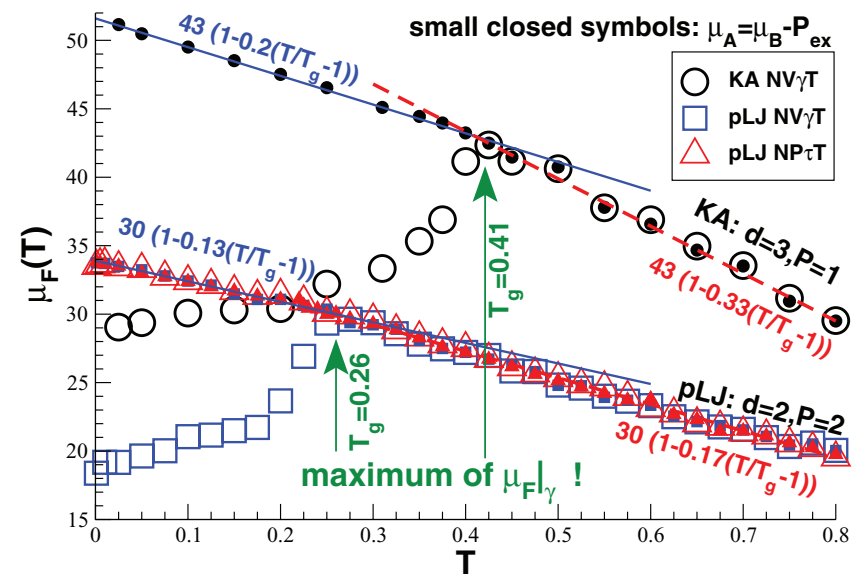

FIG. 9. Stress fluctuation $\mu_{\mathrm{F}}$ (open symbols) and affine shear elasticity $\mu_{\mathrm{A}}$ (small filled symbols) vs. temperature $T$ for both models. The indicated values have been obtained from the longest simulation runs available for a given temperature. $\mu_{\mathrm{A}}$ decays roughly linearly with $T$ as shown by bold solid lines for the low- $T$ and by dashed line for the high- $T$ regime. For constant- $\tau$ systems and for all systems with $T \geq T_{\mathrm{g}}$ we confirm that $\mu_{\mathrm{F}} \approx \mu_{\mathrm{A}}$, while $\left.\mu_{\mathrm{F}}\right|_{\gamma}$ (open spheres and squares) is seen to be non-monotonous with a maximum at $T_{\mathrm{g}}$.

In the liquid regime for $T>T_{\mathrm{g}}$ where the boundary conditions do not matter, we obtain $\left.\left.\mu_{\mathrm{F}}\right|_{\tau} \approx \mu_{\mathrm{F}}\right|_{\gamma} \approx \mu_{\mathrm{A}}$, i.e., all shear stress fluctuations decay with temperature. Below $T_{\mathrm{g}}$ the boundary constraint $(\gamma=0)$ becomes more and more relevant with decreasing $T$ which reduces the shear stress fluctuations. According to Eq. (18), $\left.\mu_{\mathrm{F}}\right|_{\gamma}$ must thus decrease with increasing $G$ as the system is further cooled down. That $\left.\mu_{\mathrm{F}}\right|_{\gamma}$ must necessarily be non-monotonous with a maximum at $T_{\mathrm{g}}$ is one of the central results of this work.

The temperature dependence of the (unscaled) shear moduli $G(T)$ is presented in Fig. 10. Data obtained by MD simulation of the KA model $\left(P \approx 1, T_{\mathrm{g}} \approx 0.41\right)$ are indicated for the $\mathrm{NV} \gamma \mathrm{T}$-ensemble (open spheres) and the NP $\gamma \mathrm{T}$ ensemble (filled spheres). All other results refer to the best

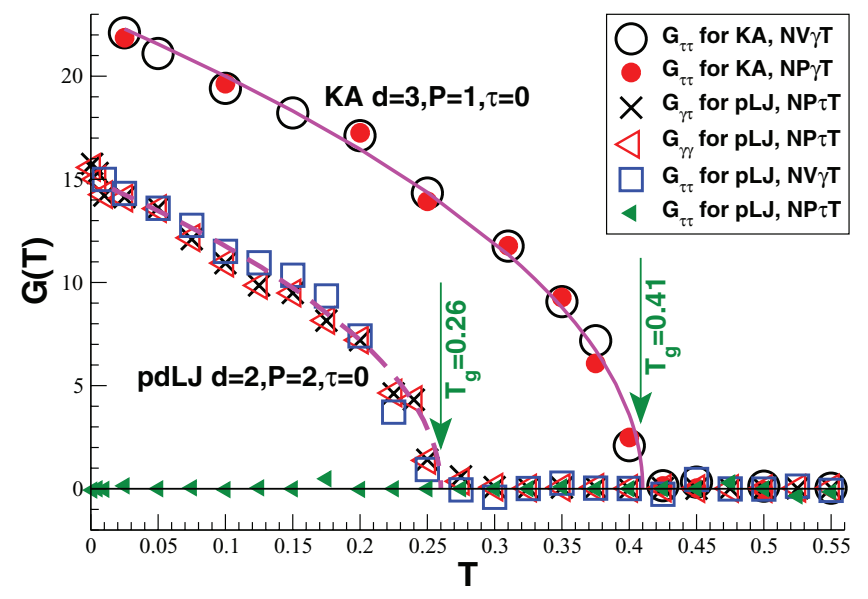

FIG. 10. Unscaled shear modulus $G$ as a function of temperature $T$ for both models. For the pLJ model the values $G_{\tau \tau}$ obtained in the $\mathrm{NV} \gamma \mathrm{T}$ ensemble (squares) are essentially identical to the moduli $G_{\gamma \tau}$ (crosses) and $G_{\gamma \gamma}$ (triangles) obtained for constant $\tau$. As shown by the small filled triangles, $G_{\tau \tau}$ vanishes in the $\mathrm{NP} \tau \mathrm{T}$-ensemble for all $T$ in agreement with Eq. (19). The solid and the dashed line indicate the cusp-singularity, Eq. (65), for, respectively, the KA model and the pLJ beads. ${ }^{30,31}$

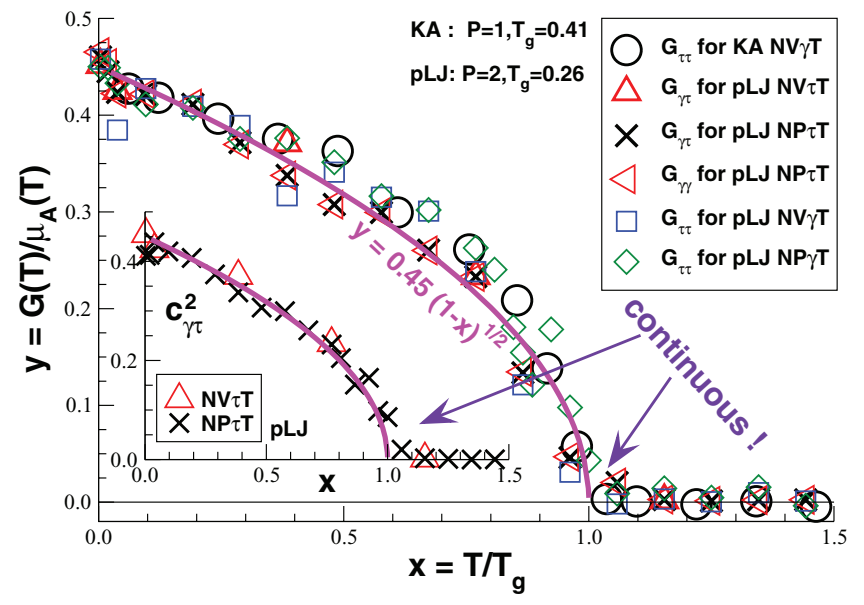

FIG. 11. Rescaled shear modulus $y=G / \mu_{\mathrm{A}}$ vs. reduced temperature $x=T / T_{\mathrm{g}}$ for the KA model at $P=1(\mathrm{NV} \gamma \mathrm{T}$-ensemble) and the $\mathrm{pLJ}$ model at $P=2$ (NV $\tau \mathrm{T}-, \mathrm{NP} \tau \mathrm{T}-, \mathrm{NV} \gamma \mathrm{T}-$, and $\mathrm{NP} \gamma \mathrm{T}$-ensembles). The bold line indicates again a continuous cusp-singularity. Inset: Squared correlation coefficient $c_{\gamma \tau}^{2}$ for $\mathrm{NV} \tau \mathrm{T}$ - and $\mathrm{NP} \tau \mathrm{T}$-ensembles.

values obtained by $\mathrm{MC}$ simulations of the $\mathrm{pLJ}$ model $(P=2$, $\left.T_{\mathrm{g}} \approx 0.26\right)$ using both local and global moves and different ensembles as indicated. For the $\mathrm{pLJ}$ model the values $G_{\tau \tau}$ obtained in the $\mathrm{NV} \gamma \mathrm{T}$ ensemble (squares) are seen to be essentially identical for all $T$ to the moduli $G_{\gamma \tau}$ (crosses) and $G_{\gamma \gamma}$ (triangles) obtained in ensembles with strain fluctuations. As shown by the small filled triangles, $G_{\tau \tau}$ vanishes in the $\mathrm{NP} \tau \mathrm{T}$ ensemble for all $T$ in agreement with Eq. (19). (We remind that the impulsive correction $\Delta \mu_{\mathrm{B}}$ has to be taken into account if $G_{\tau \tau}$ is used.) Decreasing the temperature further below $T_{\mathrm{g}}$ the shear moduli are seen to increase rapidly for both models. As shown by the solid and the dashed lines both models are well fitted by a cusp-singularity

$$
G(T) \approx g_{1}\left(1-T / T_{\mathrm{g}}\right)^{1 / 2}+g_{0} \quad \text { for } T<T_{\mathrm{g}}
$$

with fit constants $g_{1} \approx 23$ for the KA model and $g_{1} \approx 15$ for the pLJ model where we have set in both cases $g_{0}=0$. Confirming the MD simulations by Barrat et al., ${ }^{12}$ this suggests that the transition is very sharp, albeit continuous in qualitative agreement with the theoretical predictions from Refs. 23, 30, and 31. If we fit the data close to $T_{\mathrm{g}}$ with an additional off-set $g_{0}$, a slightly negative value is obtained which is not compatible with MCT. ${ }^{33,34}$ However, admittedly both the number of data points close to $T_{\mathrm{g}}$ and their precision (due to the small number of independent configurations) are yet not sufficient to rule out completely a small positive off-set.

A slightly different representation of the data is given in Fig. 11 where the reduced shear modulus $y=G(T) / \mu_{\mathrm{A}}(T)$ is plotted as a function of the reduced temperature $x=T / T_{\mathrm{g}}$ for both models. Using the independently determined glass transition temperature $T_{\mathrm{g}}$ and affine shear elasticity $\mu_{\mathrm{A}}(T)$ as scales to make the axes dimensionless, the rescaled data are found to collapse ! This is of course a remarkable and rather unexpected result considering that two different models in two different dimensions have been compared. The bold line indicates the cusp-singularity $y=0.45(1-x)^{1 / 2}$ with a prefactor which is compatible to the ones used in Eq. (65) considering the typical values of $\mu_{\mathrm{A}}(T)$ in both models. Whether 


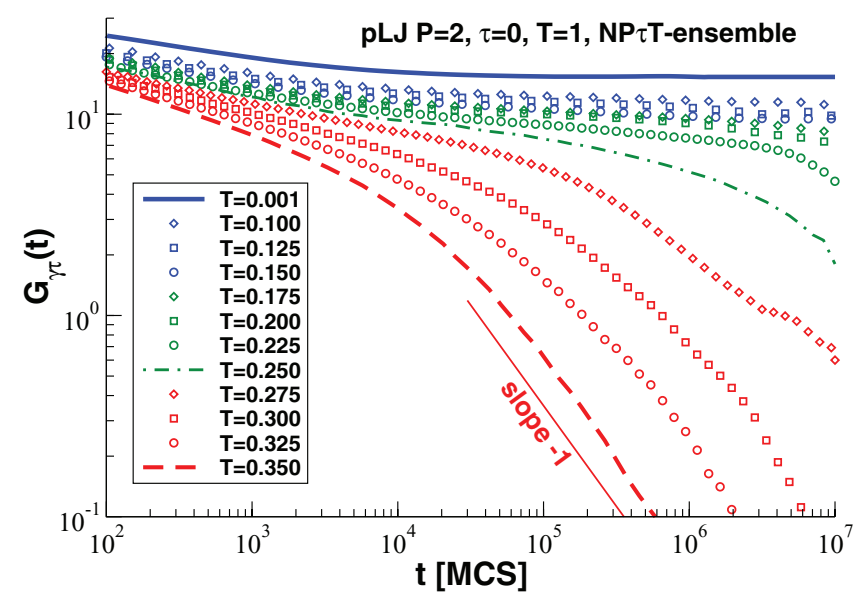

FIG. 12. Unscaled shear modulus $G_{\gamma \tau}$ as a function of sampling time $t$ in MCS obtained for the pLJ model in the NP $\tau$ T-ensemble for different temperatures as indicated. The thin line indicates the power-law slope -1 for large times in the liquid regime. A clear shoulder (on the logarithmic scales used) can only be seen below $T \approx 0.2$ and only below $T=0.01$ one observes a $t$-independent plateau over two orders of magnitude. We emphasize that we present here a sampling time effect and not a time correlation function. The same sampling time effect is observed after additional tempering.

this striking collapse is just due to some lucky coincidence or makes manifest a more general universal scaling is impossible to decide at present. No rescaling of the vertical axis is necessary for the squared correlation coefficient $c_{\gamma \tau}^{2}$ shown in the inset for two different ensembles of the $\mathrm{pLJ}$ model. The same continuous cusp-like decay (bold line) is found as for the modulus $G$. This is again consistent with the thermodynamic reasoning put forward at the end of Sec. II A, Eq. (20).

A word of caution must be placed here. The results presented in Figs. 9-11 correspond to the longest simulation runs we have at present been able to perform. Obviously, this does not mean that they correspond to the limit for asymptotically long sampling times, if the latter limit exists, or at least an intermediate plateau of respectable period of time. Focusing on the $\mathrm{pLJ}$ model in the $\mathrm{NP} \tau \mathrm{T}$-ensemble we attempt in Fig. 12 to give a tentative characterization of the sampling time dependence. We plot $G_{\gamma \tau}(t)$ as a function of $t$ for different temperatures as indicated. To make the data comparable all simulations for $T \geq 0.1$ have been performed with local MC jumps of same maximum distance $\delta r_{\max }=0.1$. As we have already seen in Fig. 4, the shear modulus decays inversely with time in the liquid regime at high temperatures. This limit is indicated by the thin line. With decreasing $T$ the (unscaled) shear modulus increases and a shoulder develops. Note that even for $T=0.25$, i.e., slightly below the glass transition temperature $T_{\mathrm{g}}=0.26$ (Fig. 2), $G_{\gamma \tau}(t)$ decays strongly with time. A more or less $t$-independent shoulder (on the logarithmic scales used) can only be seen below $T \approx 0.2$. We emphasize that we present here a sampling time effect expressing the fact that the configuration space is more and more explored with increasing $t$ and $n o t$ a time correlation function. ${ }^{10}$ Please note also that this time dependence has nothing to do with equilibration problems or ageing effects. Time translational invariance is perfectly obeyed in our simulations as we have checked by rerunning the sampling after additional tempering over at least $10^{7}$ MCS. Similar $t$-dependencies for different temperatures $T$ have also been observed for $G_{\gamma \tau}(t)$ and $G_{\tau \tau}(t)$ for the pLJ model and for $G_{\tau \tau}(t)$ for the KA model (not shown).

A different representation of the data for both models is shown in Fig. 13 where the reduced shear modulus $y=G(T) / \mu_{\mathrm{A}}(T)$ is plotted against the reduced temperature $x=T / T_{\mathrm{g}}$ for different sampling times $t$ as indicated. Similar behavior is found for both models. The shear modulus decreases with $t$ and this the more the larger the temperature $T$. For smaller temperatures the different data sets appear to approach more readily as one expects from the $t$-independent shoulder for the pLJ model in Fig. 12. As a consequence the glass transition is seen to become sharper with increasing $t$. The data approach the continuous cusp-singularity indicated by the bold line. However, it is not clear from the current data if the data converge to an at least intermediately stable behavior. Longer simulation runs are clearly necessary for both models to clarify this issue. Similar data have also been
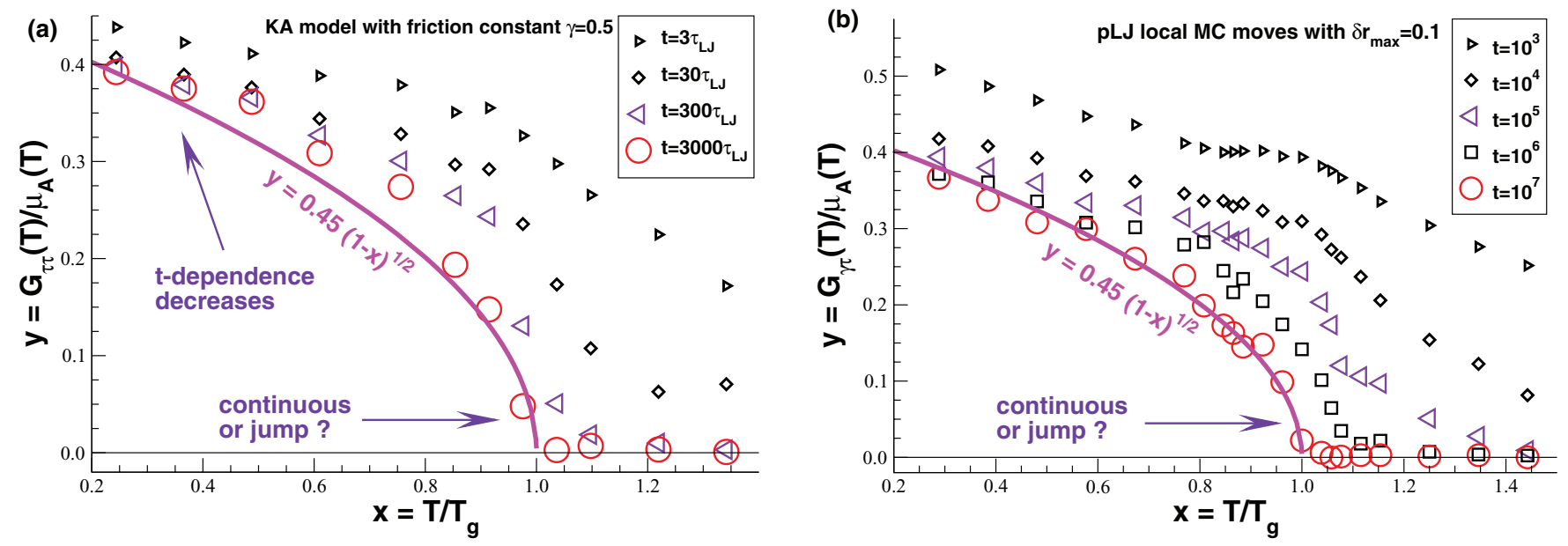

FIG. 13. Reduced shear modulus $G(T) / \mu_{\mathrm{A}}(T)$ as a function of reduced temperature $x=T / T_{\mathrm{g}}$ for different sampling times $t$ as indicated: (a) $G_{\tau \tau}(T)$ for the KA model (NV $\gamma \mathrm{T}$-ensemble), (b) $G_{\gamma \tau}(T)$ for the pLJ model (NP $\tau \mathrm{T}$-ensemble). For both models the glass transition is seen to become sharper with increasing $t$. The continuous cusp-singularity is indicated by the bold line. More points in the vicinity of $x \approx 1$ and much larger sampling times are needed in the future to decide whether the shear modulus is continuous or discontinuous at the glass transition. 
obtained for the pLJ model from the independent determination of $G_{\gamma \tau}(t)$ and $G_{\gamma \gamma}(t)$ in the NV $\tau$ T-ensemble and for $G_{\tau \tau}(t)$ in the NV $\gamma \mathrm{T}$ - and NP $\gamma \mathrm{T}$-ensembles.

\section{CONCLUSION}

Focusing on the shear modulus $G$ of two well-known glass-forming colloidal model systems in $d=3$ and $d=2$ dimensions, which we have sampled by means of MD and MC simulations, we have addressed the general question of whether the stress fluctuation method ${ }^{5-8}$ may be used through a solid-liquid transition where a microscopic displacement field ceases to be defined. Deliberately setting the experimentally motivated linear regression Eq. (6) as the fundamental definition, the shear modulus $G$ has been computed from the strain and stress fluctuations (assuming a fixed finite measurement time window $t$ ) in ensembles where either a shear strain $\gamma=0(\mathrm{NV} \gamma \mathrm{T}$ - and $\mathrm{NP} \gamma \mathrm{T}$-ensembles) or a (mean) shear stress $\tau=0$ (NV $\tau \mathrm{T}$ - and $\mathrm{NP} \tau \mathrm{T}$-ensembles) have been imposed (Fig. 1). Working at constant mean normal pressure we have compared the temperature dependence for various simple averages and fluctuations contributing to the shear modulus in different ensembles. As has been stressed in Sec. II B and Sec. II C, the stress fluctuation representation $G_{\tau \tau}$ at fixed shear strain does not rely in principle on a solid-like reference state for a displacement field of tagged particles if thermodynamics can be assumed to hold. Formally, it thus holds through the glass-transition up to the liquid regime (albeit with a trivial value $G=0$ ) as does the better known relation for the compression modulus $K$ (the Appendix). ${ }^{9}$ As emphasized in Sec. II D, impulsive corrections due to the truncation of the pair potentials have to be properly taken into account, especially at high temperatures (Fig. 4), for the precise determination of the affine shear elasticity $\mu_{\mathrm{A}}=\mu_{\mathrm{B}}$ $-P_{\text {ex }}{ }^{42}$ Confirming Eq. (10), it has been shown for the pLJ model that $G_{\tau \tau}$ at $\gamma=0$ has the same long-time behavior as the more direct observables $G_{\gamma \tau}$ and $G_{\gamma \gamma}$ obtained using the strain fluctuations at constant mean shear stress $\tau=0$. The thermodynamic relations comparing different ensembles appear thus to hold also in practice (at least within our numerical precision) through the glass transition for all $T$ and this albeit all operational definitions of $G$ are transient in the sense that they vanish for $t \rightarrow \infty$.

As predicted by general Legendre transformation, $G_{\tau \tau}$ vanishes for all temperatures $T$, if $\tau$ rather than $\gamma$ is imposed (Figs. 5, 7, and 10). The shear-stress fluctuations $\left.\mu_{\mathrm{F}}\right|_{\tau}$ are thus given by the affine response under an external load $\mu_{\mathrm{A}}$ (Fig. 9), i.e. $\left.\mu_{\mathrm{F}}\right|_{\tau}$ reduces to a simple two-point pair correlation function if pair potentials are considered as discussed in Sec. II C. The same holds above $T_{\mathrm{g}}$ for constant $\gamma$, since the boundary conditions are irrelevant for the liquid state. This symmetry with respect to the boundary conditions $(\tau \leftrightarrow \gamma)$ is broken at the glass transition for a large, but finite $t$ : at constant $\gamma$ the stress fluctuations reveal a strong non-monotonous behavior with a clear maximum at $T_{\mathrm{g}}$ (Fig. 9). The shear modulus is the order parameter characterizing this symmetry breaking. Alternatively, as we have discussed in Sec. II C, $G_{\tau \tau} / \mu_{\mathrm{A}} \equiv 1-\left.\mu_{\mathrm{F}}\right|_{\gamma} / \mu_{\mathrm{A}}$ may be seen as an order parameter comparing the ratio of the non-affine to the affine shear re- sponses. Since $\mu_{\mathrm{A}}>\left.\mu_{\mathrm{F}}\right|_{\gamma}$ for $T<T_{\mathrm{g}}$, this implies that the stress fluctuations contain higher-order correlations reducing the free energy of the system.

The increase of $G$ below $T_{\mathrm{g}}$ is reasonably fitted for both models by a continuous cusp singularity, Eq. (65), in qualitative agreement with recent theoretical predictions. ${ }^{23,30,31}$ A jump discontinuity, as suggested by mode-coupling theory ${ }^{33,34}$ and another replica theory, ${ }^{32}$ is not compatible with the currently available data shown in Figs. 10 and 11. However, as shown in Figs. 12 and 13, our data depend strongly on sampling time $t$ and the computation of larger $t$ could lead to a sharper transition. At present we believe, however, that it would be surprising if our data could be reconciled with a discontinuity at $T_{\mathrm{g}}$. It seems more likely to us that below $T_{\mathrm{g}}$, where the choice of the boundary conditions does matter as shown, the definition of the glassy shear modulus used in Refs. 32-34 may not correspond to the key operational definition $G_{\gamma \tau}$ of the shear modulus considered by us.

Future work should clearly focus on the more detailed description of the sampling time dependence. By extrapolating appropriately for the large- $t$ asymptotic behavior, this may allow to settle the theoretical debate. As emphasized above, one shortcoming of the present work is that the time-scale used in our MC simulations was slightly arbitrary and the comparison of $t$-dependent properties for different temperatures $T$ becomes thus delicate. Our MC study for the 2D soft particles should thus be recomputed with Langevin thermostat MD dynamics as used for the data of the KA model presented. The $t$-dependence of the latter model, which is a better glass former than the 2D pLJ model, ${ }^{51}$ has still to be worked out for the key operational definition $G_{\gamma \tau}$ in the NV $\tau \mathrm{T}$ - or $\mathrm{NP} \tau \mathrm{T}$ ensembles. We plan also in the near future to reconsider more carefully our previous investigation of the glass transition of polymer melts ${ }^{8}$ taking properly into account the impulsive truncation corrections and comparing the shear moduli $G_{\gamma \tau} \approx G_{\gamma \gamma}\left(\mathrm{NP} \tau \mathrm{T}\right.$-ensemble) and $G_{\tau \tau}(\mathrm{NP} \tau \mathrm{T}$-ensemble $)$ around $T_{\mathrm{g}}$. In addition, it should be rewarding to compare our shear moduli with the values obtained from the strain fluctuations at finite wavevectors in $\mathrm{NV} \gamma \mathrm{T}$-ensembles following the procedures suggested in Refs. 39 and 33.

\section{ACKNOWLEDGMENTS}

H.X. thanks the CNRS and the IRTG Soft Matter for supporting her sabbatical stay in Strasbourg and P.P. thanks the Région Alsace and the IRTG Soft Matter and F.W. the DAAD for funding. We are indebted to A. Blumen (Freiburg) and $\mathrm{H}$. Meyer, and O. Benzerara and J. Farago (all ICS, Strasbourg) for helpful discussions. We are grateful to A. Zaccone and E. M. Terentjev for communicating their recent theoretical work on disorder-assisted glass transition in amorphous solids. ${ }^{23}$

\section{APPENDIX: COMPRESSION MODULUS}

\section{Operational definitions}

We have focused in the main part of this paper on the shear modulus $G$ of our glass-forming model systems and 
the comparison of the three observables $G_{\gamma \tau}, G_{\gamma \gamma}$, and $G_{\tau \tau}$. Being isotropic the complete macroscopic elastic description of our systems only requires the numerical determination of one additional elastic modulus, the isothermal compression modulus, ${ }^{2,4,40}$

$$
K=-\left.V \frac{\partial P}{\partial V}\right|_{V}=\left.\rho \frac{\partial P}{\partial \rho}\right|_{V},
$$

characterizing the elastic response with respect to a volumetric (dilatational) strain fluctuation as shown in Fig. 1(d). As discussed in the standard textbooks, ${ }^{2,40}$ the extensive variable is here the volume $X=V=\langle\hat{V}\rangle$ and the conjugated intensive variable the negative pressure $I=-P=-\langle\hat{P}\rangle$ where $\hat{V}$ and $\hat{P}$ stand for the instantaneous values of the volume and the pressure. With these notations Eq. (A1) is obviously consistent with Eq. (12). In analogy with $G_{\gamma \tau}$ for the shear modulus $G$, the compression modulus $K$ may be determined in an $\mathrm{NP} \gamma \mathrm{T}$ - or an $\mathrm{NP} \tau \mathrm{T}$-ensemble using linear regression

$$
K_{v p} \equiv-V\langle\delta \hat{V} \delta \hat{P}\rangle /\left.\left\langle\delta \hat{V}^{2}\right\rangle\right|_{P},
$$

where the index $v p$ indicates that both the measured instantaneous volume $\hat{V}$ and pressure $\hat{P}$ have been used. The corresponding correlation coefficient is

$$
c_{v p} \equiv-\langle\delta \hat{V} \delta \hat{P}\rangle /\left.\sqrt{\left\langle\delta \hat{V}^{2}\right\rangle\left\langle\delta \hat{P}^{2}\right\rangle}\right|_{P} .
$$

Since from Eq. (13) we have $\langle\delta \hat{V} \delta \hat{P}\rangle=-k_{\mathrm{B}} T$ if $P$ is imposed, this implies that $K_{v p}$ should be identical to the better known relation ${ }^{40}$

$$
K_{v v} \equiv k_{\mathrm{B}} T V /\left\langle\delta \hat{V}^{2}\right\rangle_{P},
$$

which corresponds to the operational definition $G_{\gamma \gamma}$ discussed above. The compression modulus $K$ may of course also be determined using the pressure fluctuations in systems of imposed volume $V$ as sketched in Fig. 1(c). As (to our knowledge) first derived by Rowlinson, ${ }^{9}$ the corresponding stress fluctuation formula reads

$$
K_{p p} \equiv \eta_{\mathrm{A}}-\eta_{\mathrm{F}} \text { with } \eta_{\mathrm{F}} \equiv \beta V\left\langle\delta \hat{P}^{2}\right\rangle
$$

being the fluctuation of the total normal pressure (corresponding to $\mu_{\mathrm{F}}$ ) and $\eta_{\mathrm{A}}$ the "affine dilatational elasticity" (corresponding to $\left.\mu_{\mathrm{A}}\right)$. We shall give below a proper operational definition for $\eta_{\mathrm{A}}$. Here we only note that due to the general Legendre transformation Eq. (16) for intensive variables one knows immediately that

$$
\left.\eta_{\mathrm{F}}\right|_{V}=\left.\eta_{\mathrm{F}}\right|_{P}-K
$$

This implies by comparison with Eq. (A5) that

$$
\left.\eta_{\mathrm{A}} \stackrel{!}{=} \eta_{\mathrm{F}}\right|_{P}
$$

in analogy to Eq. (19) for the shear, i.e. $\eta_{\mathrm{A}}$ can be directly be determined from the pressure fluctuations in NP $\gamma \mathrm{T}-$ and $\mathrm{NP} \tau \mathrm{T}$-ensembles. As before for the correlation coefficient $c_{\gamma \tau}$, Eq. (20), it follows from Eq. (A7) that

$$
c_{v p}=\sqrt{K_{v v} /\left.\eta_{\mathrm{F}}\right|_{P}}=\sqrt{K / \eta_{\mathrm{A}}} .
$$

Since $c_{v p} \leq 1$, the affine dilatational elasticity sets an upper bound to the compression modulus $K$.

\section{Rowlinson's stress fluctuation formula}

We reformulate here briefly the derivation of the stress fluctuation relation $K_{p p}$ for the compression modulus $K$ of an isotropic system at imposed volume $V$ by Rowlinson. ${ }^{9}$ With $V(0)$ being the volume of the unperturbed simulation box we assume a small relative volume change

$$
\epsilon \equiv V(\epsilon) / V(0)-1
$$

with all coordinates equally deformed as, e.g.,

$$
x(0) \Rightarrow x(\epsilon)=x(0)(1+\epsilon)^{1 / d}
$$

for the $x$-coordinate in $d$-dimensions. The argument $(0)$ denotes again the reference system. Using a similar rescaling trick as for the shear modulus $G$ in Sec. II B, the interaction energy $U_{s}(\epsilon)$ of a configuration $s$ of the strained system is expressed in terms of the coordinates (state) of the unperturbed system and the explicit metric parameter $\epsilon$. Due to the volume change we have now also to take into account the ideal gas (kinetic) contributions.

We derive first the excess contribution $P_{\mathrm{ex}}$ to the total pressure $P=P_{\text {id }}+P_{\text {ex }}$ and the excess contribution $K_{\text {ex }}$ to the total compression modulus $K=K_{\mathrm{id}}+K_{\mathrm{ex}}$ valid for an arbitrary conservative potential. The general relations Eqs. (24)(27) stated above for an imposed shear strain $\gamma$ still hold with the relative volume change $\epsilon$ replacing $\gamma$. Using Eq. (26) it follows for the excess pressure that

$$
\begin{aligned}
P_{\mathrm{ex}} & =-\frac{\partial F_{\mathrm{ex}}(V)}{\partial V}=-\frac{1}{V(0)} \frac{\partial F_{\mathrm{ex}}(\epsilon)}{\partial(1+\epsilon)} \\
& =\left\langle\hat{P}_{\mathrm{ex}}\right\rangle \text { with } \hat{P}_{\mathrm{ex}} \equiv-\left.\frac{1}{V(0)} \frac{\partial U_{s}^{\prime}(\epsilon)}{\partial \epsilon}\right|_{\epsilon=0}
\end{aligned}
$$

defining the instantaneous excess pressure. ${ }^{44}$ In the second step we have taken the limit $\epsilon \rightarrow 0$ and have dropped the argument (0). The average is again evaluated using the weights of the unperturbed system, Eq. (29). Using Eqs. (25) and (27) one obtains for the compression modulus

$$
\begin{aligned}
K_{\mathrm{ex}} & =V \frac{\partial^{2} F_{\mathrm{ex}}(V)}{\partial V^{2}}=\frac{1}{V(0)} \frac{\partial^{2} F_{\mathrm{ex}}(\epsilon)}{\partial(1+\epsilon)^{2}} \\
& =\left\langle U_{s}^{\prime \prime}(\epsilon)\right\rangle / V-\beta V\left\langle\delta \hat{P}_{\mathrm{ex}}^{2}\right\rangle,
\end{aligned}
$$

which is again understood to be taken at $\epsilon=0$. The first term $\eta_{\mathrm{A}, \mathrm{ex}} \equiv\left\langle U_{s}^{\prime \prime}(\epsilon)\right\rangle / V$ in Eq. (A12) corresponds to the change of the system energy assuming an affine strain transformation for all particle positions. It gives the excess contribution $\eta_{\mathrm{A} \text {, ex }}$ to the total affine dilatational elasticity $\eta_{\mathrm{A}}=\eta_{\mathrm{A} \text {, id }}+\eta_{\mathrm{A} \text {, ex }}$. The second term corresponds to the excess contribution $\eta_{\mathrm{F} \text {, ex }}$ to the total normal pressure fluctuation $\eta_{\mathrm{F}}=\eta_{\mathrm{F} \text {, id }}+\eta_{\mathrm{F} \text {, ex }}$ mentioned in Sec. A 1. It corrects the overprediction of the affine strain contribution $\eta_{\mathrm{A}, \mathrm{ex}} \cdot{ }^{19}$

We remind that for an ideal gas the isothermal compression modulus $K_{\mathrm{id}}$ is given by the ideal gas pressure $P_{\mathrm{id}}=k_{\mathrm{B}} T \rho$ and that the ideal gas pressure fluctuation $\eta_{\mathrm{F} \text {,id }}$ $\equiv \beta V\left\langle\delta \hat{P}_{\text {id }}^{2}\right\rangle$ becomes $\left.\eta_{\mathrm{F}, \text { id }}\right|_{V}=P_{\text {id }}$ at constant volume. $^{2}$ Interestingly, using again the general Legendre transform Eq. (16) one sees that

$$
\left.\eta_{\mathrm{F}, \mathrm{id}}\right|_{P}=\left.\eta_{\mathrm{F}, \mathrm{id}}\right|_{V}+K_{\mathrm{id}}=2 P_{\mathrm{id}}
$$


for the ideal gas pressure fluctuations at imposed pressure. Due the assumed additivity of the total system Hamiltonian, the stress fluctuation formula for the total compression modulus may now be written

$$
K_{p p}=K_{\mathrm{id}}+K_{\mathrm{ex}}=P_{\mathrm{id}}+\eta_{\mathrm{A}, \mathrm{ex}}-\left.\eta_{\mathrm{F}, \mathrm{ex}}\right|_{V} .
$$

While this result is useful for constant- $V$ ensembles, especially if MC simulations are used, it is yet not in a form exactly equivalent to the general Legendre transform Eq. (A5). Since the ideal and excess pressure fluctuations are decoupled, $\left\langle\delta \hat{P}_{\text {id }} \delta \hat{P}_{\text {ex }}\right\rangle=0$, this implies $\eta_{\mathrm{F}}=\eta_{\mathrm{F} \text {, id }}+\eta_{\mathrm{F}, \text { ex }}$. One may thus rewrite Eq. (A14) as $K_{p p}=\eta_{\mathrm{A}}-\eta_{\mathrm{F}}$ with

$$
\eta_{\mathrm{A}} \equiv 2 P_{\mathrm{id}}+\eta_{\mathrm{A}, \mathrm{ex}}=\left.\eta_{\mathrm{F}, \mathrm{id}}\right|_{P}+\eta_{\mathrm{A}, \mathrm{ex}}
$$

where we have used Eq. (A13) in the second step. Assuming Eq. (A15) assures that $\eta_{\mathrm{A}}$ becomes equivalent to $\left.\eta_{\mathrm{F}}\right|_{P}$. This has the nice feature that not only the functional $K_{p p}$, Eq. (A5), gives the correct compression modulus $K$ in the $\mathrm{NV} \gamma \mathrm{T}$ and the $\mathrm{NV} \tau \mathrm{T}$ ensembles, but also that $K_{p p}$ vanishes properly if the "wrong" $\mathrm{NP} \gamma \mathrm{T}$ and $\mathrm{NP} \gamma \mathrm{T}$ ensembles are used (not shown), just as $G_{\tau \tau}$ vanishes for NV $\tau$ T- or NP $\tau$ Tensembles.

Up to now we have not taken advantage of the fact that the conservative interaction potential is assumed to be a pair potential, Eq. (39). This allows to express $P_{\mathrm{ex}}$ and $\eta_{\mathrm{A} \text {, ex }}$ in terms of simple (ensemble independent) two-point correlation functions. Equation (A10) implies that

$$
r^{2}(0) \Rightarrow r^{2}(\epsilon)=r^{2}(0)(1+\epsilon)^{2 / d} .
$$

It follows that

$$
\begin{gathered}
\frac{d r^{2}(\epsilon)}{d \epsilon} \rightarrow \frac{2}{d} r^{2}(0), \\
\frac{d^{2} r^{2}(\epsilon)}{d \epsilon^{2}} \rightarrow \frac{2(2-d)}{d^{2}} r^{2}(0),
\end{gathered}
$$

where we have taken finally for both derivatives the limit $\epsilon \rightarrow 0$. For the first two derivatives of a general function $f(r(\epsilon))$ with respect to $\epsilon$ this implies

$$
\begin{gathered}
\frac{\partial f(r(\epsilon))}{\partial \epsilon} \rightarrow \frac{1}{d} r f^{\prime}(r), \\
\frac{\partial^{2} f(r(\epsilon))}{\partial \epsilon^{2}} \rightarrow \frac{1}{d^{2}}\left(r^{2} f^{\prime \prime}(r)+r f^{\prime}(r)\right) \\
-\frac{1}{d} r f^{\prime}(r)
\end{gathered}
$$

for $\epsilon \rightarrow 0$ and dropping the argument (0) on the right-hand sides. According to the general result Eq. (A11) for the normal pressure and using Eq. (A19) one confirms for pair interactions the expected virial relation ${ }^{9,10}$

$$
\hat{P}_{\mathrm{ex}}=-\frac{1}{d V} \sum_{l} r_{l} u^{\prime}\left(r_{l}\right)
$$

for the instantaneous excess pressure where the interactions between pairs of particles $i<j$ are again labeled by an index $l$. Using Eq. (A18) the excess contribution to the affine dilatational elasticity becomes

$$
\eta_{\mathrm{A}, \mathrm{ex}}=\eta_{\mathrm{B}}+P_{\mathrm{ex}}
$$

where

$$
\eta_{\mathrm{B}} \equiv \frac{1}{d^{2} V}\left\langle\sum_{l} r_{l}^{2} u^{\prime \prime}\left(r_{l}\right)+\underline{r_{l} u^{\prime}\left(r_{l}\right)}\right\rangle
$$

is sometimes called the "hypervirial" contribution to the compression modulus. ${ }^{9,}{ }^{10}$ It corresponds to the first term indicated in Eq. (A20). By comparing Eq. (A23) with Eq. (45) it is readily seen that for isotropic systems

$$
\eta_{\mathrm{B}}=\left(1+\frac{2}{d}\right) \mu_{\mathrm{B}}-\frac{2}{d} P_{\mathrm{ex}},
$$

i.e. $\eta_{\mathrm{B}}$ and $\mu_{\mathrm{B}}$ and, hence, the affine dilatational elasticity $\eta_{\mathrm{A}}$ and affine shear elasticity $\mu_{\mathrm{A}}$ are closely related. (This justifies to call $\eta_{\mathrm{B}}$ a "Born coefficient".) Please note that Eq. (A5) or (A14) together with Eq. (A22) are perfectly consistent with the relations stated in the literature. ${ }^{7-10,22,42}$ We emphasize finally that, as discussed for the affine shear elasticity $\mu_{\mathrm{A}}$ in Sec. II B, it is inconsistent to neglect the explicit excess pressure contribution to $\eta_{\mathrm{A}}$ in Eq. (A22) but to keep the underlined term of the Born coefficient $\eta_{\mathrm{B}}$, Eq. (A23). ${ }^{52}$

\section{Numerical findings}

The affine dilatational elasticity $\eta_{\mathrm{A}}=\eta_{\mathrm{A} \text {, id }}+\eta_{\mathrm{A} \text {, ex }}$ $=2 P_{\mathrm{id}}+P_{\mathrm{ex}}+\eta_{\mathrm{B}}$ obtained for both models is presented in the inset of Fig. 14 as a function of the reduced temperature $x=T / T_{\mathrm{g}}$. We remind that for determining the Born coefficient $\eta_{\mathrm{B}}$ impulsive corrections need to be considered as discussed at the end of Sec. II D. As shown by the small filled triangles, $\eta_{\mathrm{A}}$ compares well with the total pressure fluctuation $\left.\eta_{\mathrm{F}}\right|_{P}=2 P_{\mathrm{id}}+\left.\eta_{\mathrm{F}, \text { ex }}\right|_{P}$ in an NP $\tau$ T-ensemble. The observed data collapse is expected from Eqs. (A7) and (A13). As indicated by bold and dashed lines for, respectively, the low- and high- $T$ limit $\eta_{\mathrm{A}}$ decreases essentially linearly with temperature. The coefficients of the lines (not shown) are consistent

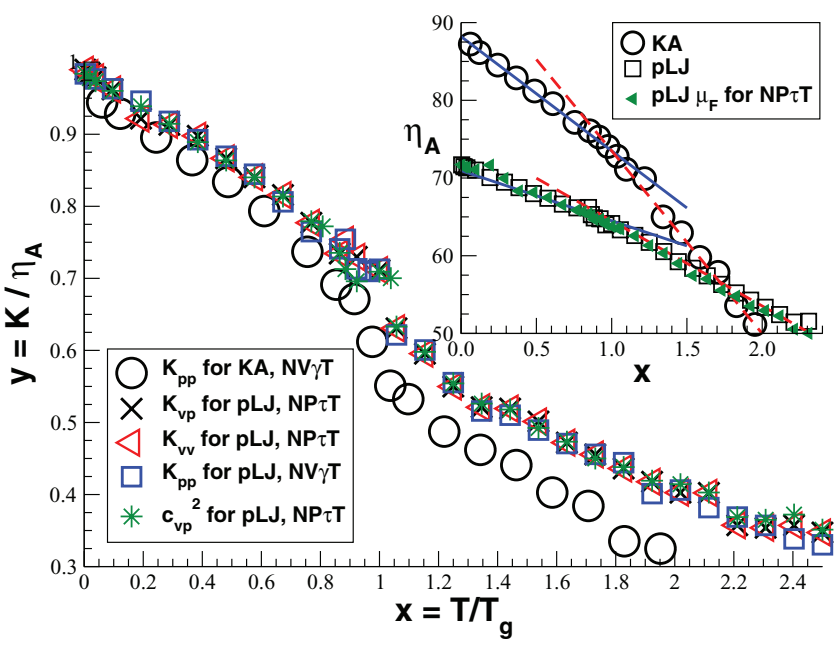

FIG. 14. Affine dilatational elasticity $\eta_{\mathrm{A}}(T)$ and compression modulus $K(T)$ for both models. Inset: $\eta_{\mathrm{A}} v s$. reduced temperature $x=T / T_{\mathrm{g}}$. The small triangles indicate $\eta_{\mathrm{F}}$ for the $\mathrm{pLJ}$ model using the $\mathrm{NP} \tau \mathrm{T}$-ensemble confirming that Eq. (A7) holds. Main panel: The Rowlinson formula $K_{p p}$, Eq. (A14), for the $\mathrm{NV} \gamma \mathrm{T}$-ensemble is given for both the KA model (spheres) and the pLJ model (squares). For the $\mathrm{pLJ}$ model we indicate in addition $K_{v p}$ (crosses), $K_{v v}$ (large triangles) and the rescaled correlation coefficient $c_{v p}^{2}$ (stars) which collapses on the other pLJ data as suggested by Eq. (A8). 
with Eq. (A24) relating $\eta_{\mathrm{A}}$ and $\mu_{\mathrm{A}}$ and the fits given in Fig. 9 for $\mu_{\mathrm{A}}$. We emphasize that the affine dilatational elasticity $\eta_{\mathrm{A}}$ (and thus $\mu_{\mathrm{A}}$ ) is not only larger for the KA model but, more importantly that, is also increases more rapidly with decreasing $T$ than the $\mathrm{pLJ}$ model. The main panel of Fig. 14 shows the best long-time values of the compression modulus $K$ for both models. The vertical axis is rescaled by $\eta_{\mathrm{A}}$. The rescaled stress fluctuation formula, Eq. (A14), for the $\mathrm{NV} \gamma \mathrm{T}$-ensemble is given for both models. For the pLJ model we also indicate $K_{v p}$ (crosses) and $K_{v v}$ (large triangles) for the $\mathrm{NP} \tau \mathrm{T}$-ensemble. No rescaling with $\eta_{\mathrm{A}}$ is necessary for the squared correlation coefficient $c_{v p}^{2}$ (stars) also presented. A perfect collapse of all $\mathrm{pLJ}$ data is found confirming the equivalence of all operational definitions used. For both models we observe a strong increase of $K / \eta_{\mathrm{A}}$ around $x \approx 1$ with decreasing temperature. Interestingly, the rescaled data of both models is similar but not identical. The mentioned increase around $x \approx 1$ is clearly more pronounced for the KA model. Note that if the unscaled $K$ is directly plotted against $T$, this effect becomes even stronger (not shown) consistently with the scaling of $\eta_{\text {A }}$ presented in the inset. We note finally that for very low temperatures we observe for both models that the ratio $K / \eta_{\mathrm{A}}=c_{\gamma \tau}^{2}$ approaches unity, i.e., the compression modulus is essentially dominated by the affine response. We remind that this is different for the reduced shear modulus for both models which is observed to approach $G / \mu_{\mathrm{A}}$ $\approx 1 / 2$ in the low- $T$ limit (Fig. 11), i.e. the shear stress fluctuations $\left.\mu_{\mathrm{F}}\right|_{\gamma}$ are more relevant than the pressure fluctuations $\left.\eta_{\mathrm{F}}\right|_{V}$.

${ }^{1}$ M. Born and K. Huang, Dynamical Theory of Crystal Lattices (Clarendon, Oxford, 1954)

${ }^{2}$ P. M. Chaikin and T. C. Lubensky, Principles of Condensed Matter Physics (Cambridge University Press, 1995).

${ }^{3}$ D. C. Wallace, in Solid State Physics: Advances in Research and Applications, edited by H. Ehrenreich, F. Seitz, and D. Turnbull (Academic, New York/London, 1970), Vol. 25, p. 300.

${ }^{4}$ L. D. Landau and E. M. Lifshitz, Theory of Elasticity (Pergamon, 1959).

${ }^{5}$ D. R. Squire, A. C. Holt, and W. G. Hoover, Physica 42, 388 (1969).

${ }^{6}$ J. F. Lutsko, J. Appl. Phys. 65, 2991 (1989).

${ }^{7}$ D. Frenkel and B. Smit, Understanding Molecular Simulation - From Algorithms to Applications, 2nd ed. (Academic, San Diego, 2002).

${ }^{8}$ B. Schnell, H. Meyer, C. Fond, J. Wittmer, and J. Baschnagel, Eur. Phys. J. E 34, 97 (2011).

${ }^{9}$ J. S. Rowlinson, Liquids and Liquid Mixtures (Butterworths Scientific, London, 1959).

${ }^{10} \mathrm{M}$. Allen and D. Tildesley, Computer Simulation of Liquids (Oxford University Press, Oxford, 1994).

${ }^{11}$ J. R. Ray, M. C. Moody, and A. Rahman, Phys. Rev. B 32, 733 (1985).

${ }^{12}$ J.-L. Barrat, J.-N. Roux, J.-P. Hansen, and M. L. Klein, Europhys. Lett. 7, 707 (1988).

${ }^{13}$ O. Farago and Y. Kantor, Phys. Rev. E 61, 2478 (2000).

${ }^{14}$ J. P. Wittmer, A. Tanguy, J.-L. Barrat, and L. Lewis, Europhys. Lett. 57, 423 (2002).

${ }^{15}$ A. Tanguy, J. P. Wittmer, F. Leonforte, and J.-L. Barrat, Phys. Rev. B 66, 174205 (2002)

${ }^{16}$ A. Tanguy, F. Leonforte, J. P. Wittmer, and J.-L. Barrat, Appl. Surf. Sci. 226, 282 (2004).

${ }^{17}$ F. Léonforte, R. Boissière, A. Tanguy, J. P. Wittmer, and J.-L. Barrat, Phys. Rev. B 72, 224206 (2005).

${ }^{18}$ C. Maloney and A. Lemaitre, Phys. Rev. Lett. 93, 195501 (2004).

${ }^{19} \mathrm{~J}$.-L. Barrat, in Computer Simulations in Condensed Matter Systems: From Materials to Chemical Biology, edited by M. Ferrario, G. Ciccotti, and K. Binder (Springer, Berlin/Heidelberg, 2006), Vol. 704, pp. 287-307.

${ }^{20}$ K. van Workum and J. de Pablo, Phys. Rev. E 67, 011505 (2003).
${ }^{21}$ K. Yoshimoto, T. Jain, K. van Workum, P. Nealey, and J. de Pablo, Phys. Rev. Lett. 93, 175501 (2004).

${ }^{22}$ N. Schulmann, H. Xu, H. Meyer, P. Polińska, J. Baschnagel, and J. P. Wittmer, Eur. Phys. J. E 35, 93 (2012).

${ }^{23}$ A. Zaccone and E. Terentjev, preprint arXiv:1212.2020 (2013).

${ }^{24}$ F. Léonforte, A. Tanguy, J. P. Wittmer, and J.-L. Barrat, Phys. Rev. Lett. 97, 055501 (2006).

${ }^{25} \mathrm{M}$. Tsamados, A. Tanguy, C. Goldenberg, and J.-L. Barrat, Phys. Rev. E 80, 026112 (2009).

${ }^{26}$ G. Papakonstantopoulos, R. Riggleman, J. L. Barrat, and J. J. de Pablo, Phys. Rev. E 77, 041502 (2008).

${ }^{27}$ D. P. Landau and K. Binder, A Guide to Monte Carlo Simulations in Statistical Physics (Cambridge University Press, Cambridge, 2000).

${ }^{28}$ S. Ulrich, X. Mao, P. Goldbart, and A. Zippelius, Europhys. Lett. 76, 677 (2006).

${ }^{29}$ G. Parisi and F. Zamponi, Rev. Mod. Phys. 82, 789 (2010).

${ }^{30} \mathrm{H}$. Yoshino and M. Mézard, Phys. Rev. Lett. 105, 015504 (2010).

${ }^{31}$ H. Yoshino, J. Chem. Phys. 136, 214108 (2012).

${ }^{32}$ G. Szamel and E. Flenner, Phys. Rev. Lett. 107, 105505 (2011).

${ }^{33}$ C. Klix, F. Ebert, F. Weysser, M. Fuchs, G. Maret, and P. Keim, Phys. Rev. Lett. 109, 178301 (2012).

${ }^{34} \mathrm{~W}$. Götze, Complex Dynamics of Glass-Forming Liquids: A ModeCoupling Theory (Oxford University Press, Oxford, 2009).

${ }^{35}$ S. J. Plimpton, J. Comput. Phys. 117, 1 (1995).

${ }^{36}$ T. Mason and D. Weitz, Phys. Rev. Lett. 75, 2770 (1995).

${ }^{37}$ E. del Gado and W. Kob, J. Non-Newtonian Fluid Mech. 149, 28 (2008).

${ }^{38}$ A. Zilman, J. Kieffer, F. Molino, G. Porte, and S. A. Safran, Phys. Rev. Lett. 91, 015901 (2003).

${ }^{39}$ S. Senguptal, P. Nielaba, M. Rao, and K. Binder, Phys. Rev. E 61, 1072 (2000).

${ }^{40} \mathrm{H}$. B. Callen, Thermodynamics and an Introduction to Thermostatistics (Wiley, New York, 1985).

${ }^{41}$ We have computed the static correlation function $\beta V\langle\delta \hat{\gamma} \delta \hat{\tau}\rangle_{\tau}$ for a broad range of temperatures and pressures for both $\mathrm{NV} \tau \mathrm{T}$ - and $\mathrm{NP} \tau \mathrm{T}$-ensembles. Converging rapidly to unity, this confirms that Eq. (8) holds to a high accuracy well up into the liquid regime.

${ }^{42}$ H. Xu, J. Wittmer, P. Polińska, and J. Baschnagel, Phys. Rev. E 86, 046705 (2012).

${ }^{43}$ For the simplicity of the notation we assume that $X$ is not the inner energy $U$. For a more thorough systematic theoretical description it is necessary to define the "entropic intensive variable" $J \equiv \partial S(X) / \partial X$ with $S(X)$ being the entropy. ${ }^{40}$ If $X \neq U$, one has $J=-I / T .{ }^{40}$ These entropic intensive variables are used (for good reasons) in Ref. 45. Note that expressing Eq. (15) in terms of $I$, rather than in terms of $J$, changes the signs.

${ }^{44}$ There is considerable freedom for defining $\hat{I}$ as long as the average $I$ does not change. The experimentally natural choice for $\hat{I}=\hat{\tau}$ is given by the instantaneous horizontal force (per surface) on the top wall in Fig. 1(a). For theory and simulation, Eq. (28) or Kirkwood's formula, Eq. (40), are more suitable for $\hat{\tau}$, but by no means unique.

${ }^{45}$ J. L. Lebowitz, J. K. Percus, and L. Verlet, Phys. Rev. 153, 250 (1967).

${ }^{46}$ M. Abramowitz and I. A. Stegun, Handbook of Mathematical Functions (Dover, New York, 1964).

${ }^{47}$ R. Zwanzig and R. Mountain, J. Chem. Phys. 43, 4464 (1965).

${ }^{48}$ J. Hansen and I. McDonald, Theory of Simple Liquids (Academic, New York, 1986).

${ }^{49}$ W. Kob and H. C. Andersen, Phys. Rev. E 52, 4134 (1995).

${ }^{50}$ For the quench of the configurations sufficiently small values for $\delta \gamma_{\max }$ for a shear strain altering MC move and for $\delta V_{\max }$ for a volume change have been chosen such that the acceptance rate of both MC steps remain reasonable at the lowest temperatures $(T<0.1)$.

${ }^{51}$ Similar values for $T_{\mathrm{g}}$ have also been obtained for both models following the standard diagnostics described in the literature ${ }^{12,49}$ of various dynamic properties such as the scaling of the mean-square displacements, the self-diffusion coefficient or the Fourier transformed density autocorrelation function in the low-wavevector limit. From the analysis of these dynamical properties it is seen that, compared to the KA model, the pLJ model is a more gradual glass-former qualitatively similar to the purely repulsive soft-sphere model studied in Ref. 12.

${ }^{52} \mathrm{As}$ for the shear stress fluctuations at constant $\tau$ in Sec. II C, it is possible to demonstrate by integration by parts, Eq. (48), that Eq. (A7) holds with $\eta_{\mathrm{A}}$ being given by Eqs. (A15) and (A23). Using the Legendre transform Eq. (A6) one thus immediately obtains Rowlinson's pressure fluctuation formula, Eq. (A5). 